TRANSACTIONS OF THE

AMERICAN MATHEMATICAL SOCIETY

Volume 362, Number 4, April 2010, Pages 1721-1750

S 0002-9947(09)04895-8

Article electronically published on November 18, 2009

\title{
DOUBLE VOGAN DIAGRAMS AND SEMISIMPLE SYMMETRIC SPACES
}

\author{
MENG-KIAT CHUAH AND JING-SONG HUANG
}

\begin{abstract}
A Vogan diagram is a set of involution and painting on a Dynkin diagram. It selects a real form, or equivalently an involution, from a complex simple Lie algebra. We introduce the double Vogan diagram, which is two sets of Vogan diagrams superimposed on an affine Dynkin diagram. They correspond to pairs of commuting involutions on complex simple Lie algebras, and therefore provide an independent classification of the simple locally symmetric pairs.
\end{abstract}

\section{INTRODUCTION}

A semisimple symmetric space is a homogeneous manifold $G / H$, where $G$ is a semisimple Lie group with an involution $\sigma$, and $H$ is a closed subgroup satisfying $\left(G^{\sigma}\right)_{0} \subset H \subset G^{\sigma}$. These spaces were classified by Berger [3]. By ignoring the different covering spaces, this amounts to the pair $(\mathfrak{g}, \mathfrak{h})$, where $\mathfrak{g}$ is a real semisimple Lie algebra, and $\mathfrak{h}$ is the fixed points of an involution on $\mathfrak{g}$. Such pairs $(\mathfrak{g}, \mathfrak{h})$ are known as the (semisimple) locally symmetric pairs. There exists a Cartan involution $\theta$ on $\mathfrak{g}$ which commutes with $\sigma$. By complex linear extension, we obtain an ordered pair of commuting involutions $(\theta, \sigma)$ on the complexification $\mathfrak{g}^{\mathbf{c}}$. Conversely, given commuting involutions $(\theta, \sigma)$ on a complex semisimple Lie algebra, there exists a $\theta$ stable and $\sigma$-stable real form $\mathfrak{g}$ such that $\theta$ is a Cartan involution on $\mathfrak{g}$. We conclude that the locally symmetric pairs are equivalent to the ordered pairs of commuting involutions on a complex semisimple Lie algebra. Helminck [8] classified such pairs $(\theta, \sigma)$, and furthermore distinguishes the inner and outer involutions. Huang [9] also classified the locally symmetric pairs by square quadruplets. The main purpose of this paper is to use the Dynkin diagrams to provide another combinatorial classification of the locally symmetric pairs, or equivalently the commuting involutions on complex semisimple Lie algebras. We shall call them double Vogan diagrams. Our diagrams provide rich algebraic information on the roots of $\mathfrak{g}$. Since $\sigma$ commutes with $\theta$, it preserves the Cartan decomposition $\mathfrak{g}=\mathfrak{k}+\mathfrak{p}$, where $\mathfrak{k}=\mathfrak{g}^{\theta}$. Thus we also study the extension problem of involutions $\sigma$ from $\mathfrak{k}$ to $\mathfrak{g}$. Based on Berger's classification, Oshima and Sekiguchi [12] determined the restricted root systems of

Received by the editors February 9, 2007.

2000 Mathematics Subject Classification. Primary 17B20, 53C35.

Key words and phrases. Vogan diagram, locally symmetric pair, Dynkin diagram, simple Lie algebra, involution.

The first author was supported in part by the National Center for Theoretical Sciences and the National Science Council of Taiwan.

The second author was supported in part by research grants from the Research Grant Council of HKSAR and the National Natural Science Foundation of China.

(C)2009 American Mathematical Society Reverts to public domain 28 years from publication 1721 
locally symmetric pairs. We note that the works of Oshima-Sekiguchi and Helminck have used the maximally split Cartan subalgebras of $\mathfrak{g}$, while our present work uses the maximally compact Cartan subalgebras.

We now describe our project in better detail. To avoid discussion on the simple factors, we always assume that $\mathfrak{g}^{\mathbf{c}}$ is simple. The superscript $\mathbf{c}$ always denotes complexification. Once and for all, we ignore the trivial cases $\theta=1$ (g compact), $\theta=\sigma$ and $\sigma=1$. Let $\operatorname{inv}(\cdot)$ denote "involutions on".

Let $\mathfrak{g}$ be a real simple Lie algebra with Cartan involution $\theta$ and Cartan decomposition $\mathfrak{k}+\mathfrak{p}$. Let $\mathrm{D}$ be the Dynkin diagram of $\mathfrak{g}^{\mathbf{c}}$. A Vogan diagram [11, Ch.VI-8] is a diagram involution on $\mathrm{D}$, such that the vertices fixed by the involution are painted white or black. The Vogan diagrams represent real forms of $\mathfrak{g}^{\mathbf{c}}$, so some of them represent $\mathfrak{g}$. We next introduce the affine Vogan diagrams, which reveal the algebraic properties of $\mathfrak{g}=\mathfrak{k}+\mathfrak{p}$ better than the Vogan diagrams.

A diagram automorphism on $\mathrm{D}$ of order $r \in\{1,2,3\}$ leads to the affine Dynkin diagram $\mathrm{D}^{r}$, as introduced by Kac [10, [7, p.503]. Each vertex $\alpha$ of $\mathrm{D}^{r}$ is associated to a canonical positive integer coefficient $m_{\alpha}$ (see (2.2) $)$. An affine Vogan diagram is an affine Dynkin diagram $\mathrm{D}^{r}$ whose vertices are painted white or black, such that

$$
r \sum_{\text {black }} m_{\alpha}=2 .
$$

Due to this condition, we shall ignore $\mathrm{D}^{3}$ from now on.

Theorem 1.1 (Kac [10, 7]). An affine Vogan diagram represents a real simple Lie algebra $\mathfrak{g}=\mathfrak{k}+\mathfrak{p}$, where the white vertices form the Dynkin diagram of $\mathfrak{k}^{\mathbf{c}}$, and the black vertices are the lowest weights of the $\mathfrak{k}^{\mathbf{c}}$-representation on $\mathfrak{p}^{\mathbf{c}}$. Conversely, every real simple Lie algebra is represented by a unique affine Vogan diagram in this way.

The conjugate classes of real forms correspond to the conjugate classes of involutions $\theta$ on the complex simple Lie algebra; the correspondence being $\theta$ restricts to be a Cartan involution on the real form. Therefore, a Vogan diagram or affine Vogan diagram also represents an involution. Theorem 1.1 is a special case of Kac's theorem on order $m$ automorphisms on complex simple Lie algebras [7, Chap. X, Thms. 5.15, 5.16], where $m=2$ in Theorem 1.1.

There are several advantages of affine Vogan diagrams over Vogan diagrams. Affine Vogan diagrams entirely reveal $\mathfrak{k}$ and $\mathfrak{p}$ by the white and black vertices. Also, by using the twisted diagrams $\mathrm{D}^{2}$, there is no need for diagram involution. Another advantage is the uniqueness (up to diagram automorphism) of the affine Vogan diagram which represents $\mathfrak{g}$. On the contrary, $\mathfrak{g}$ can be represented by several Vogan diagrams, despite ways (Theorem 2.1) to help reduce redundant ones.

Suppose that $(\theta, \sigma)$ is a pair of commuting involutions on $\mathfrak{g}^{\mathbf{c}}$. By Theorem 1.1. we use an affine Vogan diagram to represent the decomposition $\mathfrak{g}^{\mathbf{c}}=\mathfrak{k}^{\mathbf{c}}+\mathfrak{p}^{\mathbf{c}}$ with respect to $\theta$. The white vertices of an affine Vogan diagram form the Dynkin diagram of $\mathfrak{k}^{\mathbf{c}}$, so we denote it by $\mathrm{D}_{\mathfrak{k}}$. Since $\sigma$ preserves $\mathfrak{k}^{\mathbf{c}}$ and $\mathfrak{p}^{\mathbf{c}}$, we can represent $\sigma \in \operatorname{inv}\left(\mathfrak{k}^{\mathfrak{c}}\right)$ by a second set of Vogan diagrams on $\mathrm{D}_{\mathfrak{k}}$ (see Corollary 2.2). Namely, $\sigma \in \operatorname{inv}\left(\mathfrak{k}^{\mathbf{c}}\right)$ is represented by a diagram involution on the white vertices, such that the vertices fixed by the involution are uncircled or circled. A vertex is uncircled (resp. circled) if $\sigma=1$ (resp. $\sigma=-1$ ) on its root vectors. We use circling instead of painting to distinguish the action of $\sigma$ from $\theta$. The Vogan diagram of $\sigma \in \operatorname{inv}\left(\mathfrak{k}^{c}\right)$ is non-trivial, as $\sigma \neq 1$ on $\mathfrak{k}^{\mathbf{c}}$. This is because if $1 \in \operatorname{inv}\left(\mathfrak{k}^{\mathbf{c}}\right)$ extends to $\operatorname{inv}\left(\mathfrak{g}^{\mathbf{c}}\right)$, then 
the extension can only be 1 or $\theta$, and we have already agreed to rule out these trivial cases. Strictly speaking, the Vogan diagram on $D_{\mathfrak{k}}$ only represents $\sigma \in \operatorname{inv}\left(\mathfrak{k}^{\mathbf{c}} / \mathfrak{z}^{\mathbf{c}}\right)$, where $\mathfrak{z}^{\mathbf{c}}$ is the center of $\mathfrak{k}^{\mathbf{c}}$. But if $\sigma$ indeed extends to $\operatorname{inv}\left(\mathfrak{g}^{\mathbf{c}}\right)$, then its value on $\mathfrak{z}^{\mathbf{c}}$ is forced (see Proposition 2.4). So we may regard the Vogan diagram on $D_{\mathfrak{k}}$ as representing $\sigma \in \operatorname{inv}\left(\mathfrak{k}^{\mathbf{c}}\right)$.

Next we study the extension of $\sigma \in \operatorname{inv}\left(\mathfrak{k}^{\mathbf{c}}\right)$ to $\operatorname{inv}\left(\mathfrak{g}^{\mathbf{c}}\right)$. By Proposition 2.4, we may as well assume that the diagram involution on $D_{\mathfrak{k}}$ extends to the entire affine Vogan diagram, since this is necessary (but not sufficient) to represent a Lie algebra involution which preserves $\mathfrak{k}^{\mathbf{c}}$ and $\mathfrak{p}^{\mathbf{c}}$. By a diagram involution on the affine Dynkin diagram, we shall always mean an involution on the diagram which preserves the vertex colors.

We let $\sigma$ denote the involution on $\mathfrak{g}$ and $\mathfrak{g}^{\mathbf{c}}$, as well as the involution on the diagram and roots. The Lie algebra involution maps the root space of $\alpha$ to the root space of $\sigma \alpha$. There are four possibilities for a vertex $\alpha$ :

$$
\left\{\begin{array}{l}
\text { an uncircled vertex } \alpha \text { fixed by } \sigma ; \\
\text { a circled vertex } \alpha \text { fixed by } \sigma ; \\
\text { distinct vertices }\{\alpha, \sigma \alpha\} \text { which are adjacent; } \\
\text { distinct vertices }\{\alpha, \sigma \alpha\} \text { which are not adjacent. }
\end{array}\right.
$$

Let $\mathcal{O}$ denote the $\sigma$-orbits of vertices of the second and the third type in (1.2). Namely,

$$
\mathcal{O}=\{\text { circled vertices }\} \cup\{\sigma \text {-orbit of two adjacent vertices }\} .
$$

Definition 1.2. A double Vogan diagram is an affine Vogan diagram on $\mathrm{D}^{r}$ with a diagram involution, such that the vertices fixed by the involution are uncircled or circled, and such that $r \sum_{\mathcal{O}} m_{\alpha}$ is even. We say that a double Vogan diagram represents a locally symmetric pair $\left(\mathfrak{g}, \mathfrak{g}^{\sigma}\right)$ if:

(a) the underlying affine Vogan diagram represents $\mathfrak{g}$;

(b) the Vogan diagram on the white vertices represents $\sigma \in \operatorname{inv}\left(\mathfrak{k}^{\mathrm{c}}\right)$;

(c) the diagram involution and circling on the black vertices represent $\sigma$ on $\mathfrak{p}^{\mathbf{c}}$.

In general, we say that a diagram represents a Lie algebra involution $\sigma$ if there are canonical root vectors $X_{\alpha}$ (see (2.7)) such that $\sigma X_{\alpha}=X_{\sigma \alpha}$ if $\alpha \neq \sigma \alpha$, and $\sigma X_{\alpha}= \pm X_{\alpha}$ if $\alpha=\sigma \alpha$, where the sign is + or - depending on whether vertex $\alpha$ is uncircled or circled.

The following is the main theorem of this paper. It leads to the diagrammatic classification of the locally symmetric pairs.

Theorem 1.3 (Main Theorem). Every double Vogan diagram represents a locally symmetric pair $(\mathfrak{g}, \mathfrak{h})$ uniquely up to conjugation. Conversely, every locally symmetric pair is represented by a double Vogan diagram.

The double Vogan diagrams help to explain the extension of $\mathfrak{k}^{\mathbf{c}}$-involutions to $\mathfrak{g}^{\mathbf{c}}$-involutions. They lead to the following corollary.

Corollary 1.4 (Extension Theorem).

(a) Each $\sigma \in \operatorname{inv}\left(\mathfrak{k}^{\mathbf{c}}\right)$ can have 0,1 or 2 extensions (up to conjugation) to $\operatorname{inv}\left(\mathfrak{g}^{\mathbf{c}}\right)$.

(b) If $\mathfrak{g}$ is of Hermitian type, then $\sigma \in \operatorname{inv}\left(\mathfrak{k}^{\mathbf{c}}\right)$ is extendable to $\operatorname{inv}\left(\mathfrak{g}^{\mathbf{c}}\right)$ provided the diagram involution of $\sigma$ is trivial.

(c) If $\mathfrak{g}$ is of non-equal rank type, then every $\sigma \in \operatorname{inv}\left(\mathfrak{k}^{\mathfrak{c}}\right)$ has exactly two nonconjugate extensions to $\operatorname{inv}\left(\mathfrak{g}^{\mathbf{c}}\right)$. 
Z. D. Yan studied the above extension problem and obtained similar results, though we can only find the reference in a Chinese book [13] published by Nankai University. Our approach in this paper is simpler and entirely different from that of Yan.

According to Theorem 1.3, every double Vogan diagram represents a unique (up to conjugate class) locally symmetric pair. However, there may be several diagrams which represent the same locally symmetric pair. In this case we say that the diagrams are equivalent. In Theorem 5.1 , we shall describe the method to check for equivalent diagrams. Consequently, we obtain an independent classification of locally symmetric pairs via a bijective correspondence with the equivalence classes of double Vogan diagrams. We shall draw the diagrams to provide a complete list of locally symmetric pairs.

While we match the diagrams with the locally symmetric pairs, we have referred to [9] for relevant information. However, [9, pp. 116-119] contains some misprints and mistakes on the list of exceptional locally symmetric pairs. Therefore, we shall take this opportunity to make corrections to the list.

Our diagrams have several advantages over previous classifications of locally symmetric pairs. They describe the locally symmetric pairs just as Dynkin diagrams do for the complex simple Lie algebras. They describe the lowest weights of the $\mathfrak{k}^{\mathbf{c}}$ representation on $\mathfrak{p}^{\mathbf{c}}$, and the $\theta$ - and $\sigma$-actions on the roots of $\mathfrak{k}$ and $\mathfrak{p}$. They reveal the types of $\mathfrak{g}$, such as whether $\mathfrak{g}$ is of equal or non-equal rank type, and of Hermitian or non-Hermitian type (see (2.5)). They also reveal locally symmetric pairs which are associate, as the diagrams coincide on $D_{\mathfrak{k}}$. We shall discuss elsewhere their applications, such as the signatures of pseudo-Riemannian structures, the existence of pseudo-Hermitian structures, and the ranks and types of $\mathfrak{g}, \mathfrak{h}, \mathfrak{k}, \mathfrak{h} \cap \mathfrak{k}$.

The sections are organized as follows. In Section 2, we establish the notation and recall some structure theory of simple Lie algebras. In Section 3, we prove Theorem 1.3. In Section 4, we prove Corollary 1.4. In Section 5, we develop the method to check for equivalent diagrams, leading to Theorem [5.1. In Section 6, we provide several examples to illustrate our ideas. In Section 7, we apply Theorem 1.3 to list the double Vogan diagrams and their locally symmetric pairs, where Theorem 5.1 helps to avoid equivalent diagrams. In Section 8, we eliminate the mistakes in 9 , pp. 116-119] and provide the correct list of exceptional locally symmetric pairs.

\section{Preliminaries on Simple Lie algebras}

In this section, we recall some background material on Lie algebras and set up the notation for later sections. Recall that a Vogan diagram is a diagram involution on a Dynkin diagram, such that the vertices fixed by the involution are painted white and black 11.

Theorem 2.1 (Borel and de Siebenthal [4, 11, Thm. 6.96]). Every Vogan diagram represents a real simple Lie algebra. Conversely, every real simple Lie algebra is represented by a Vogan diagram with at most one black vertex $\alpha$, where $m_{\alpha}=1$ or $m_{\alpha}=2$.

The integers $m_{\alpha}$ are explained in (2.2). This theorem can be modified easily for real semisimple Lie algebras, where the condition on $m_{\alpha}$ is imposed on each connected component of the Dynkin diagram.

Let $L$ be a complex simple Lie algebra, with a Cartan subalgebra $H \subset L$. Let $\Phi^{s} \subset \Phi \subset H^{*}$ be the simple system and root system. The root spaces are denoted 
by $L_{\alpha}$; thus $L=H+\sum_{\Phi} L_{\alpha}$. Let $\mathrm{D}$ be the Dynkin diagram of $L$, and let the vertices of $\mathrm{D}$ represent $\Phi^{s}$.

We say that a Vogan diagram on $\mathrm{D}$ represents $\sigma \in \operatorname{inv}(L)$ if the diagram involution (also denoted by $\sigma$ ) satisfies $\sigma L_{\alpha}=L_{\sigma \alpha}$ for all $\alpha \in \Phi^{s}$, and $\sigma=1$ (resp. $\sigma=-1$ ) on $L_{\alpha}$ if $\alpha$ is a white (resp. black) vertex.

Corollary 2.2. Let $\sigma$ be an involution on a complex semisimple Lie algebra L. Then $\sigma$ can be represented by a Vogan diagram with at most one black vertex $\alpha$, where $m_{\alpha}=1$ or $m_{\alpha}=2$.

Proof. Let $\sigma$ be an involution on a complex semisimple Lie algebra $L$. We can find a real form $\mathfrak{g}$ of $L$ such that $\sigma$ restricts to a Cartan involution on $\mathfrak{g}$ : Regarding $L$ as real semisimple, it has a Cartan involution $\theta$ which commutes with $\sigma$ [11, Thm. 6.16]. Its fixed point set $L^{\theta}$ is a compact real form of $L$. Then the fixed point set of $\sigma \theta$, namely $\mathfrak{g}=L^{\sigma \theta}$, is a real form of $L$ with Cartan involution $\sigma$.

By Theorem 2.1, $\mathfrak{g}$ is represented by a Vogan diagram on D. This is constructed by choosing a $\sigma$-stable maximally compact Cartan subalgebra $\mathfrak{h}$ of $\mathfrak{g}$, so that the Cartan subalgebra $\mathfrak{h} \otimes \mathbf{C}$ of $L$ has a $\sigma$-stable simple system $\Phi^{s}$ [11, Ch.VI-8]. Let $\sigma$ denote both the involution on $L$ as well as the diagram involution on $\mathrm{D}$. Then $\sigma$ permutes the simple root spaces, and $\sigma L_{\alpha}=L_{\sigma \alpha}$ for all $\alpha \in \Phi^{s}$. Let $\mathfrak{g}=\mathfrak{k}+\mathfrak{p}$ be the Cartan decomposition arising from $\sigma$. The white (resp. black) vertices of the Vogan diagram are the simple roots in $\mathfrak{k}^{\mathbf{c}}\left(\right.$ resp. $\mathfrak{p}^{\mathbf{c}}$ ), so $\sigma=1$ (resp. $\sigma=-1$ ) on their root spaces. Theorem 2.1 also says that the Vogan diagram can be chosen to have at most one black vertex $\alpha$, where $m_{\alpha}=1$ or $m_{\alpha}=2$. This completes the proof.

Let aut $(\cdot)$ denote "automorphisms on". Let $f \in \operatorname{aut}(\mathrm{D})$. This leads naturally to $f \in \operatorname{aut}(\Phi)$, and hence to $f \in \operatorname{aut}(L)$ by $f L_{\alpha}=L_{f \alpha}$, and $f=1$ on $L_{\alpha}$ if $f \alpha=\alpha$.

Let $r$ be the order of $f$, so $r \in\{1,2,3\}$. Let $\mathbf{Z}_{r}$ be the finite abelian group of integers modulo $r$. We obtain a $\mathbf{Z}_{r}$-grading $L=\sum_{a \in \mathbf{Z}_{r}} L^{a}$, where $L^{a}$ are the eigenvectors of $f \in \operatorname{aut}(L)$ with eigenvalue $\left(\exp \frac{2 \pi i}{r}\right)^{a}$. The reductive subalgebra $L^{0}$ acts on each $L^{a}$ by $\left[L^{0}, L^{a}\right] \subset L^{a}$. A Cartan subalgebra of $L^{0}$ is $H^{0}=H \cap L^{0}$. Define

$$
\Pi \cup\{\varphi\} \subset \Delta \subset\left(H^{0}\right)^{*}
$$

by letting $\Pi \subset \Delta \subset\left(H^{0}\right)^{*}$ be the restriction of $\Phi^{s} \subset \Phi \subset H^{*}$ to $H^{0}$, and $\varphi \in\left(H^{0}\right)^{*}$ be the lowest weight of the $L^{0}$-representation on $L^{1}$. So $\Pi$ is a simple system of $L^{0}$. The affine Dynkin diagram $\mathrm{D}^{r}$ is the diagram whose vertices are $\Pi \cup\{\varphi\}$, and whose edges follow the usual law with root lengths and angles. Note that if $r=1$, then $\Phi^{s}=\Pi$ and $\Phi=\Delta$, and $\varphi$ is the lowest root. Then $D^{1}$ is just an extension of $\mathrm{D}$ with extra vertex $\varphi$.

Since $\Pi$ is a basis of $\left(H^{0}\right)^{*}$, the set $\Pi \cup\{\varphi\}$ is linearly dependent. Define

$$
m_{\alpha}
$$

to be the canonical positive integers such that $\sum_{\Pi \cup\{\varphi\}} m_{\alpha} \alpha=0$ and such that $\left\{m_{\alpha}\right\}$ have no non-trivial common factor. We use $m_{\alpha}$ in Theorem 2.1 by regarding $\mathrm{D}$ as a subdiagram of $\mathrm{D}^{1}$. For the reader's convenience, we now provide the diagrams $\mathrm{D}^{1}$ and $\mathrm{D}^{2}$ along with $m_{\alpha}$. 
Affine Dynkin Diagrams D ${ }^{1}$

\begin{tabular}{|c|c|}
\hline$\overbrace{1}^{1}-\cdots-\overbrace{1}^{\infty}$ & $\overbrace{1}^{B_{n}^{1}}$ \\
\hline$C_{n}^{1}$ & 10 \\
\hline$E_{6}^{1}$ & $E_{7}^{1}$ \\
\hline$E_{8}^{1}$ & 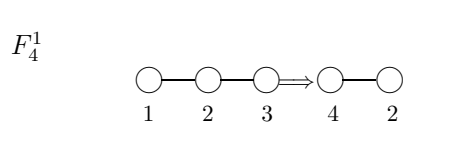 \\
\hline$G_{2}^{1} \underset{1}{\bigcirc}-\underset{2}{\rightleftharpoons} \underset{3}{\rightleftharpoons}$ & \\
\hline
\end{tabular}

Affine Dynkin Diagrams D ${ }^{2}$

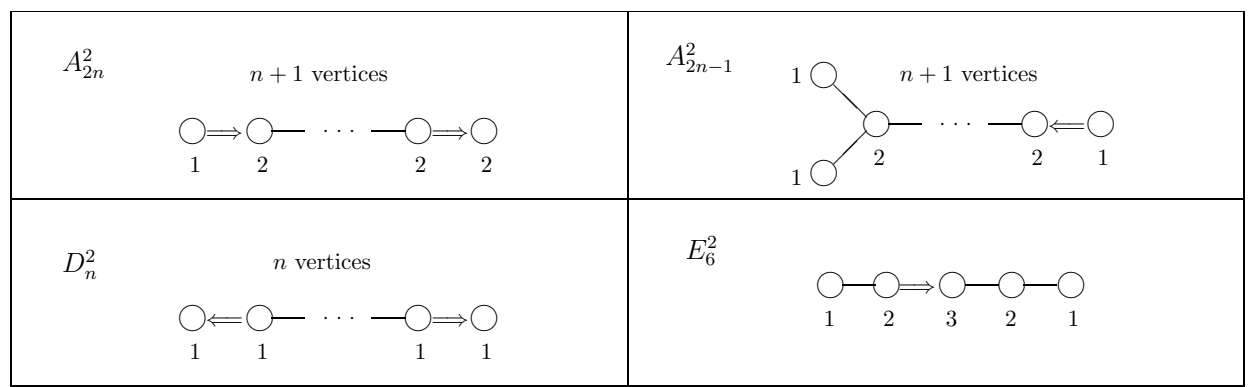

Take $L$ to be $\mathfrak{g}^{\mathbf{c}}$, and $H^{0}$ a Cartan subalgebra of $\mathfrak{k}^{\mathbf{c}}$. So $\Delta \subset\left(H^{0}\right)^{*}$ are the roots of $\mathfrak{k}^{\mathrm{c}}$, and we have

$$
\mathfrak{g}^{\mathbf{c}}=H^{0}+\sum_{\Delta} \mathfrak{g}_{\alpha}^{\mathbf{c}}
$$

Since $H^{0}$ is equal to or smaller than a Cartan subalgebra of $\mathfrak{g}^{\mathbf{c}}$, the root space $\mathfrak{g}_{\alpha}^{\mathbf{c}}$ can be 1-dimensional or 2-dimensional. We write

$$
\mathfrak{g}_{\alpha}^{\mathbf{c}}=\left(\mathfrak{g}_{\alpha}^{\mathbf{c}} \cap \mathfrak{k}^{\mathbf{c}}\right)+\left(\mathfrak{g}_{\alpha}^{\mathbf{c}} \cap \mathfrak{p}^{\mathbf{c}}\right)=\mathfrak{k}_{\alpha}^{\mathbf{c}}+\mathfrak{p}_{\alpha}^{\mathbf{c}} .
$$


If $\mathfrak{g}_{\alpha}^{\mathbf{c}}$ is 1-dimensional, then it is either $\mathfrak{k}_{\alpha}^{\mathbf{c}}$ or $\mathfrak{p}_{\alpha}^{\mathbf{c}}$. If $\mathfrak{g}_{\alpha}^{\mathbf{c}}$ is 2-dimensional, then each of $\mathfrak{k}_{\alpha}^{\mathrm{c}}$ and $\mathfrak{p}_{\alpha}^{\mathrm{c}}$ is 1-dimensional. From now on, when we talk about roots, root spaces or weight spaces, we always refer to $\alpha \in \Delta, \mathfrak{g}_{\alpha}^{\mathbf{c}}, \mathfrak{k}_{\alpha}^{\mathbf{c}}$ or $\mathfrak{p}_{\alpha}^{\mathbf{c}}$ discussed above.

The construction of $\mathrm{D}^{1}, \mathrm{D}^{2}$ and $m_{\alpha}$ leads to the definition of affine Vogan diagrams by (1.1). An advantage of the affine Vogan diagrams over the Vogan diagrams is that each real simple Lie algebra $\mathfrak{g}$ is represented by a unique affine Vogan diagram. On the contrary, despite the conditions on $m_{\alpha}$ of Theorem 2.1, there can be distinct Vogan diagrams which represent $\mathfrak{g}$. Examples include $\mathfrak{s o}(4,2 n-4) \cong$ $\mathfrak{s o}(2 n-4,4)$ and $\mathfrak{e}_{6(2)}$.

We may replace $r \sum_{\text {black }} m_{\alpha}=2$ of (1.1) by the weaker condition

$$
r \sum_{\text {black }} m_{\alpha} \text { is even. }
$$

This leads to additional diagrams, and they represent involutions which are conjugate to the ones resulting from (1.1). This can be regarded as the affine version of Theorem 2.1, or can be seen from explicit algorithms [6].

The condition $r \sum_{\text {black }} m_{\alpha}=2$ on $\mathrm{D}^{r}$ leads to three possibilities:

$$
\left\{\begin{array}{l}
\text { (a) } \quad r=1, \text { two black vertices } \gamma, \delta \text { with } m_{\gamma}=m_{\delta}=1 ; \\
\text { (b) } r=1, \text { one black vertex } \gamma \text { with } m_{\gamma}=2 ; \\
\text { (c) } \quad r=2, \text { one black vertex } \gamma \text { with } m_{\gamma}=1 .
\end{array}\right.
$$

They correspond precisely to the following types of $\mathfrak{g}$. Let $\mathfrak{z}$ denote the center of $\mathfrak{k}$. (1) Equal rank type: We say that $\mathfrak{g}$ is of equal rank type if $\mathfrak{g}$ and $\mathfrak{k}$ have the same rank. We use an affine Vogan diagram on $\mathrm{D}^{1}$. Here $\varphi$ is the lowest root of $\Pi$. These Lie algebras are further divided as follows.

(1a) Hermitian type: Geometrically, this means that the Riemannian symmetric space $G / K$ is Hermitian. The adjoint $\mathfrak{k}^{\mathbf{c}}$-representation on $\mathfrak{p}^{\mathbf{c}}$ has two irreducible components, so its affine Vogan diagram belongs to (2.5) (a). The center $\mathfrak{z}$ of $\mathfrak{k}$ is 1-dimensional.

(1b) Non-Hermitian type: Geometrically, this means that the Riemannian symmetric space $G / K$ is not Hermitian. The adjoint $\mathfrak{k}^{\mathbf{c}}$-representation on $\mathfrak{p}^{\mathbf{c}}$ is irreducible. So its affine Vogan diagram belongs to (2.5) (b). Here $\mathfrak{k}$ is semisimple, so $\mathfrak{z}=0$.

(2) Non-equal rank type: We say that $\mathfrak{g}$ is of non-equal rank type if $\mathfrak{g}$ has greater rank than $\mathfrak{k}$. Its affine Vogan diagram belongs to (2.5) (c). Here $\mathfrak{k}$ is semisimple, so $\mathfrak{z}=0$.

Now consider $\sigma \in \operatorname{inv}\left(\mathfrak{g}^{\mathbf{c}}\right)$ which commutes with $\theta$. It preserves $\mathfrak{k}^{\mathbf{c}}$ and $\mathfrak{p}^{\mathbf{c}}$, so we can represent $\sigma \in \operatorname{inv}\left(\mathfrak{k}^{\mathbf{c}}\right)$ by a Vogan diagram on the white vertices $D_{\mathfrak{k}}$. This is a diagram involution on $\mathrm{D}_{\mathfrak{k}}$ whose fixed vertices are uncircled or circled, depending on whether $\sigma=1$ or $\sigma=-1$ on its root space. We use "circling" instead of "painting" to distinguish $\sigma$ from $\theta$. We choose the Vogan diagram of $\sigma \in \operatorname{inv}\left(\mathfrak{k}^{\mathbf{c}}\right)$ by Corollary 2.2. so that it has at most one circled white vertex on each connected component of $\mathrm{D}_{\mathfrak{k}}$.

Our study of commuting involutions $(\theta, \sigma)$ on a complex simple Lie algebra is carried out by the following three steps.

$$
\left\{\begin{array}{l}
\text { Step 1: Describe } \mathfrak{g}=\mathfrak{k}+\mathfrak{p} \text { by an affine Vogan diagram. } \\
\text { Step 2: Describe } \sigma \in \operatorname{inv}\left(\mathfrak{k}^{\mathbf{c}} / \mathfrak{z}^{\mathbf{c}}\right) \text { or } \sigma \in \operatorname{inv}\left(\mathfrak{k}^{\mathbf{c}}\right) \text { on } D_{\mathfrak{k}} \\
\text { Step 3: Study the extension of } \sigma \in \operatorname{inv}\left(\mathfrak{k}^{\mathbf{c}}\right) \text { to } \operatorname{inv}\left(\mathfrak{g}^{\mathbf{c}}\right)
\end{array}\right.
$$


Steps 1 and 2 have been achieved by the above discussions. Strictly speaking, the Vogan diagram on $D_{\mathfrak{k}}$ only represents $\operatorname{inv}\left(\mathfrak{k}^{\mathbf{c}} / \mathfrak{z}^{\mathbf{c}}\right)$ and not $\operatorname{inv}\left(\mathfrak{k}^{\mathbf{c}}\right)$. However, as we shall see in Proposition 2.4, if $\sigma \in \operatorname{inv}\left(\mathfrak{k}^{\mathbf{c}}\right)$ extends to $\operatorname{inv}\left(\mathfrak{g}^{\mathbf{c}}\right)$, then its value on $\mathfrak{z}^{\mathbf{c}}$ is forced. So we can regard the Vogan diagram on $D_{\mathfrak{k}}$ as representing $\sigma \in \operatorname{inv}\left(\mathfrak{k}^{\mathbf{c}}\right)$ for our purpose. Step 3 will be discussed in the next section.

Definition 2.3. An almost double Vogan diagram is an affine Vogan diagram with a diagram involution, such that the vertices fixed by the involution are uncircled or circled.

By Definition 1.2 an almost double Vogan diagram is weaker than a double Vogan diagram only because $r \sum_{\mathcal{O}} m_{\alpha}$ may not be even.

Proposition 2.4. Let $\sigma \in \operatorname{inv}\left(\mathfrak{k}^{\mathbf{c}}\right)$ be represented by a Vogan diagram on $\mathrm{D}_{\mathfrak{k}}$. If $\sigma$ extends to an involution on $\mathfrak{g}^{\mathbf{c}}$, then the Vogan diagram on $\mathrm{D}_{\mathfrak{k}}$ extends to an almost double Vogan diagram. Furthermore, if $\mathfrak{g}$ is of Hermitian type, then one has that

(a) the diagram involution preserves the black vertices implies $\sigma=1$ on $\mathfrak{z}^{\mathbf{c}}$; and

(b) the diagram involution interchanges the two black vertices implies $\sigma=-1$ on $\mathfrak{z}^{\mathrm{c}}$.

Proof. Suppose that $\sigma \in \operatorname{inv}\left(\mathfrak{k}^{\mathbf{c}}\right)$ extends to an involution on $\mathfrak{g}^{\mathbf{c}}$. It preserves the Killing form of $\mathfrak{g}^{\mathbf{c}}$, and $\mathfrak{p}^{\mathbf{c}}$ is the orthogonal complement of $\mathfrak{k}^{\mathbf{c}}$ with respect to the Killing form. So $\sigma$ preserves $\mathfrak{k}^{\mathbf{c}}$ and $\mathfrak{p}^{\mathbf{c}}$. Therefore, it sends the lowest weight spaces of $\mathfrak{p}^{\mathbf{c}}$ to extreme weight spaces of $\mathfrak{p}^{\mathbf{c}}$. But since $\sigma$ on $\mathfrak{k}^{\mathbf{c}}$ is represented by a Vogan diagram on $\mathrm{D}_{\mathfrak{k}}$, it preserves the simple roots $\Pi$ of $\mathfrak{k}^{\mathbf{c}}$. Therefore, $\sigma$ preserves the lowest weight spaces of $\mathfrak{p}^{\mathbf{c}}$. Consequently, it preserves the black vertices of the affine Vogan diagram. So it is represented by an almost double Vogan diagram.

It remains to prove parts (a) and (b). We may assume that $\mathfrak{g}$ is of Hermitian type, since otherwise $\mathfrak{z}=0$. The only vertices of $D^{1}$ which do not annihilate $\mathfrak{z}$ are the two black vertices, because $\mathfrak{z}$ is the center of $\mathfrak{k}$. Given a root $\alpha$, let $\hat{\alpha}$ be its coroot, namely $\alpha=B(\hat{\alpha}, \cdot)$, where $B$ is the Killing form. We may choose the root vectors $X_{\alpha} \in \mathfrak{g}_{\alpha}^{\mathbf{c}}$ from the Cartan-Chevalley basis of $\mathfrak{g}^{\mathbf{c}}$, and they satisfy

$$
\left[X_{\alpha}, X_{-\alpha}\right]=\hat{\alpha}, \text { and } \sigma X_{\alpha}=X_{\sigma \alpha}
$$

whenever $\sigma \alpha \neq \alpha$ [11, Ch.VI-8].

For part (a), suppose that a black vertex $\gamma$ is fixed by the diagram involution. Depending on whether vertex $\gamma$ is circled or not, we have $\sigma= \pm 1$ on $\mathfrak{p}_{\gamma}^{\mathbf{c}}$ and $\mathfrak{p}_{-\gamma}^{\mathbf{c}}$. The signs on $\mathfrak{p}_{\gamma}^{\mathbf{c}}$ and $\mathfrak{p}_{-\gamma}^{\mathbf{c}}$ are the same; hence $\left[\sigma X_{\gamma}, \sigma X_{-\gamma}\right]=\left[X_{\gamma}, X_{-\gamma}\right]$. Pick $0 \neq Z \in \mathfrak{z}^{\mathbf{c}}$. By (2.7),

$$
\gamma(Z)=B\left(Z,\left[X_{\gamma}, X_{-\gamma}\right]\right)=B\left(\sigma Z,\left[\sigma X_{\gamma}, \sigma X_{-\gamma}\right]\right)=B\left(\sigma Z,\left[X_{\gamma}, X_{-\gamma}\right]\right)=\gamma(\sigma Z) .
$$

Since $\gamma$ does not annihilate $Z$, this implies that $\sigma=1$ on $\mathfrak{z}^{\mathbf{c}}$. This proves (a).

For part (b), suppose that the diagram involution interchanges the two black vertices $\delta$ and $\gamma$. Pick $0 \neq Z \in \mathfrak{z}^{\text {c }}$. By (2.7),

$$
\delta(Z)=B\left(Z,\left[X_{\delta}, X_{-\delta}\right]\right)=B\left(\sigma Z,\left[\sigma X_{\delta}, \sigma X_{-\delta}\right]\right)=B\left(\sigma Z,\left[X_{\gamma}, X_{-\gamma}\right]\right)=\gamma(\sigma Z) .
$$

Since $\sum_{\mathrm{D}^{1}} m_{\alpha} \alpha=0$, and since $\delta, \gamma$ are the only vertices of $\mathrm{D}^{1}$ which do not annihilate $Z$, we conclude that $\delta(Z)+\gamma(Z)=0$. It then follows from (2.8) that $\sigma Z=-Z$. This proves (b).

In the above paragraph and also elsewhere, we clearly let $\sum_{\mathrm{D}_{\mathfrak{k}}}$ and $\sum_{\mathrm{D}^{r}}$ denote summation over their respective vertices. 


\section{Double Vogan diagrams}

In this section, we prove Theorem 1.3. The idea is to perform Step 3 of (2.6). Namely, by Definition 2.3 and Proposition 2.4 we assume that we already have an almost double Vogan diagram. We shall show that $r \sum_{\mathcal{O}} m_{\alpha}$ is even if and only if it represents an involution on $\mathfrak{g}^{\mathbf{c}}$.

By (2.3), $\Delta$ are the $\mathfrak{k}^{\mathrm{c}}$-roots with simple roots $\Pi$, and their root spaces are $\mathfrak{g}_{\alpha}^{\mathbf{c}}$. The vertices of $\mathrm{D}^{r}$ are $\Pi \cup\{\varphi\}$. Let $\overline{\mathcal{O}}=(\Pi \cup\{\varphi\}) / \sigma$ denote the $\sigma$-orbits of vertices, as given in (1.2).

From the almost double Vogan diagram, we obtain $\sigma \in \operatorname{inv}(\Delta)$. Suppose that $\sigma \in \operatorname{inv}\left(\mathfrak{k}^{\mathbf{c}}\right)$ extends to $\operatorname{inv}\left(\mathfrak{g}^{\mathbf{c}}\right)$. If $\alpha \in \Delta$ is fixed by $\sigma$, then $\mathfrak{g}_{\alpha}^{\mathbf{c}}$ are eigenvectors of $\sigma$, with eigenvalue denoted by

$$
\mathrm{e}(\alpha) \in\{ \pm 1\}
$$

It is sometimes more convenient to deal with $\overline{\mathcal{O}}$ than with the roots, so we introduce the following notation. Write

$$
\alpha+\beta \in \Delta ; \alpha \in \overline{\mathcal{O}}, \beta \in \overline{\mathcal{O}} \text { or } \beta \in \Delta
$$

to denote addition of all the roots involved. For example if $\alpha=\{\gamma, \sigma \gamma\}$ and $\beta$ is a root, then $\alpha+\beta=\gamma+\sigma \gamma+\beta$. We also write

$$
E \in \mathfrak{g}_{\alpha}^{\mathbf{c}}, \operatorname{ad}_{E}: \mathfrak{g}^{\mathbf{c}} \longrightarrow \mathfrak{g}^{\mathbf{c}} ; \alpha \in \overline{\mathcal{O}}
$$

In (3.3), if $\alpha=\{\gamma, \sigma \gamma\}$, then $E$ denotes an ordered pair $(X, Y) \in \mathfrak{g}_{\gamma}^{\mathbf{c}} \times \mathfrak{g}_{\sigma \gamma}^{\mathbf{c}}$, and $\operatorname{ad}_{E}=\operatorname{ad}_{X} \cdot \operatorname{ad}_{Y}$.

Proposition 3.1. Let $\beta \in \Delta$ be fixed by $\sigma$, and let $\alpha \in \overline{\mathcal{O}}$. Suppose that $\alpha+\beta$ is a root.
(a) If $\alpha$ is an uncircled vertex, then $\mathrm{e}(\alpha+\beta)=\mathrm{e}(\beta)$.
(b) If $\alpha$ is a circled vertex, then $\mathrm{e}(\alpha+\beta)=-\mathrm{e}(\beta)$.
(c) If $\alpha$ is a $\sigma$-orbit of adjacent vertices, then $\mathrm{e}(\alpha+\beta)=-\mathrm{e}(\beta)$.
(d) If $\alpha$ is a $\sigma$-orbit of non-adjacent vertices, then $\mathrm{e}(\alpha+\beta)=\mathrm{e}(\beta)$.

Proof. Here e is as defined in (3.1), and $\alpha+\beta$ is as defined in (3.2). The assumptions of the proposition imply that $\alpha+\beta$ is a root fixed by $\sigma$, so $\mathrm{e}(\alpha+\beta)$ makes sense.

For part (a), an element of $\mathfrak{g}_{\alpha+\beta}^{\mathbf{c}}$ is of the form $[E, X]$, where $E \in \mathfrak{g}_{\alpha}^{\mathbf{c}}$ and $X \in \mathfrak{g}_{\beta}^{\mathbf{c}}$. Then $\sigma[E, X]=[\sigma E, \sigma X]=[E, \mathrm{e}(\beta) X]=\mathrm{e}(\beta)[E, X]$. Part (b) is just as trivial.

For part (c), let $\alpha=\{\gamma, \sigma \gamma\}$. Pick a non-zero element of $\mathfrak{g}_{\alpha+\beta}^{\mathbf{c}}=\mathfrak{g}_{\gamma+\sigma \gamma+\beta}^{\mathrm{c}}$ which is of the form $Z=[[E, \sigma E], X]$, where $E \in \mathfrak{g}_{\gamma}^{\mathbf{c}}$ and $X \in \mathfrak{g}_{\beta}^{\mathbf{c}}$. Then

$$
\sigma Z=\sigma[[E, \sigma E], X]=[[\sigma E, E], \sigma X]=-\mathrm{e}(\beta)[[E, \sigma E], X]=-\mathrm{e}(\beta) Z .
$$

For part (d), let $\alpha=\{\gamma, \sigma \gamma\}$ again. Pick a non-zero element of $\mathfrak{g}_{\alpha+\beta}^{\mathbf{c}}=\mathfrak{g}_{\gamma+\sigma \gamma+\beta}^{\mathbf{c}}$ which is given by $Z=[\sigma E,[E, X]]$, where $E \in \mathfrak{g}_{\gamma}^{\mathbf{c}}$ and $X \in \mathfrak{g}_{\beta}^{\mathbf{c}}$. Then

$$
\begin{aligned}
\sigma Z & =\sigma[\sigma E,[E, X]] \\
& =\mathrm{e}(\beta)[E,[\sigma E, X]] \\
& =\mathrm{e}(\beta)[\sigma E,[E, X]] \quad \text { by the Jacobi identity and }[E, \sigma E]=0 \\
& =\mathrm{e}(\beta) Z
\end{aligned}
$$

This proves part $(\mathrm{d})$, and the proposition follows.

Let $\mathcal{O} \subset \overline{\mathcal{O}}$ be as given in (1.3). In the following corollary, a member of $\overline{\mathcal{O}}$ may appear several times in the summation $\alpha_{1}+\ldots+\alpha_{n}$. Similarly, the same 
member is counted with multiplicity towards $\sharp\left(\left\{\alpha_{1}, \ldots, \alpha_{n}\right\} \cap \mathcal{O}\right)$, where $\sharp(\cdot)$ denotes "the number of elements of". Here $\alpha_{1}+\ldots+\alpha_{i}$ is as defined in (3.2). By abuse of notation, we allow $\alpha_{1} \in \Delta$ when $\alpha_{1}$ is a pair of adjacent vertices. Namely $\alpha_{1}=\{\gamma, \sigma \gamma\} \in \Delta$ means $\gamma+\sigma \gamma \in \Delta$.

Corollary 3.2. Let $\alpha=\alpha_{1}+\ldots+\alpha_{n}$, where $\alpha_{i} \in \overline{\mathcal{O}}$ and $\alpha_{1}+\ldots+\alpha_{i} \in \Delta$ for all $i=1, \ldots, n$. Then $\sigma=1$ (resp. $\sigma=-1$ ) on $\mathfrak{g}_{\alpha}^{\mathbf{c}}$ if and only if $\sharp\left(\left\{\alpha_{1}, \ldots, \alpha_{n}\right\} \cap \mathcal{O}\right)$ is even (resp. odd).

Proof. Note that $\sigma$ fixes $\alpha_{1}+\ldots+\alpha_{i}$, so e $\left(\alpha_{1}+\ldots+\alpha_{i}\right)$ makes sense. Since each $\alpha_{i} \in \overline{\mathcal{O}}$ belongs to one of the four types of $\sigma$-orbits in Proposition 3.1, there exist $\epsilon_{1}, \ldots, \epsilon_{n} \in\{ \pm 1\}$ such that

$$
\begin{aligned}
\mathrm{e}\left(\alpha_{1}+\ldots+\alpha_{n}\right) & =\epsilon_{n} \mathrm{e}\left(\alpha_{1}+\ldots+\alpha_{n-1}\right) \\
& =\epsilon_{n-1} \epsilon_{n} \mathrm{e}\left(\alpha_{1}+\ldots+\alpha_{n-2}\right) \\
& =\ldots=\epsilon_{1} \ldots \epsilon_{n} .
\end{aligned}
$$

By Proposition 3.1, the number of -1 's which occur in $\left\{\epsilon_{1}, \ldots, \epsilon_{n}\right\}$ is $\sharp\left(\left\{\alpha_{1}, \ldots, \alpha_{n}\right\} \cap\right.$ $\mathcal{O})$. If this quantity is even, then $\mathrm{e}(\alpha)=1$, so $\sigma=1$ on $\mathfrak{g}_{\alpha}^{\mathrm{c}}$. Similarly, if this quantity is odd, then $\mathrm{e}(\alpha)=-1$, so $\sigma=-1$ on $\mathfrak{g}_{\alpha}^{\mathbf{c}}$. This proves the corollary.

The almost double Vogan diagram on $\mathrm{D}^{r}$ restricts to a Vogan diagram on $\mathrm{D}_{\mathfrak{k}}$, and hence defines $\sigma_{\mathfrak{k}} \in \operatorname{inv}\left(\mathfrak{k}^{\mathbf{c}}\right)$. The almost double Vogan diagram also defines an action $\sigma_{\mathfrak{p}}$ on the lowest weight spaces $\mathfrak{p}_{\gamma}^{\mathbf{c}}$ by $\sigma_{\mathfrak{p}}=1$ (resp. $\sigma_{\mathfrak{p}}=-1$ ) on the weight spaces of the uncircled (resp. circled) vertices $\gamma$; and also $\sigma_{\mathfrak{p}}: \mathfrak{p}_{\gamma}^{\mathbf{c}} \longrightarrow \mathfrak{p}_{\sigma \gamma}^{\mathbf{c}} \longrightarrow \mathfrak{p}_{\gamma}^{\mathbf{c}}$ by (2.7) when the diagram involution interchanges the two black vertices $\{\gamma, \sigma \gamma\}$. Then $\sigma_{\mathfrak{p}}$ extends to a vector space involution $\sigma_{\mathfrak{p}} \in \operatorname{inv}\left(\mathfrak{p}^{\mathbf{c}}\right)$ by $\sigma_{\mathfrak{p}}[X, Y]=\left[\sigma_{\mathfrak{k}} X, \sigma_{\mathfrak{p}} Y\right]$ for all $X \in \mathfrak{k}^{\mathbf{c}}$ and $Y \in \mathfrak{p}_{\gamma}^{\mathbf{c}}$. By letting $\sigma_{\mathfrak{k}}$ and $\sigma_{\mathfrak{p}}$ act on $\mathfrak{k}^{\mathbf{c}}$ and $\mathfrak{p}^{\mathbf{c}}$ separately, we obtain a vector space involution $\sigma_{\mathfrak{k}} \oplus \sigma_{\mathfrak{p}}$ on $\mathfrak{g}^{\mathbf{c}}=\mathfrak{k}^{\mathbf{c}}+\mathfrak{p}^{\mathbf{c}}$.

Proposition 3.3. We obtain a Lie algebra involution $\sigma_{\mathfrak{k}} \oplus \sigma_{\mathfrak{p}} \in \operatorname{inv}\left(\mathfrak{k}^{\mathbf{c}}+\mathfrak{p}^{\mathbf{c}}\right)$ if and only if $r \sum_{\mathcal{O}} m_{\alpha}$ is even.

Proof. Given $X, Y \in \mathfrak{p}^{\mathbf{c}}$, we can let $\sigma_{\mathfrak{k}}$ act on $[X, Y]$, or use the Lie bracket and send it to $\left[\sigma_{\mathfrak{p}} X, \sigma_{\mathfrak{p}} Y\right]$. So for $\sigma_{\mathfrak{k}} \oplus \sigma_{\mathfrak{p}}$ to be a Lie algebra involution on $\mathfrak{g}^{\mathbf{c}}$, it is necessary and sufficient that

$$
\sigma_{\mathfrak{k}}[X, Y]=\left[\sigma_{\mathfrak{p}} X, \sigma_{\mathfrak{p}} Y\right]
$$

for all $X, Y \in \mathfrak{p}^{\mathbf{c}}$. For simplicity, write $f[X, Y]=\left[\sigma_{\mathfrak{p}} X, \sigma_{\mathfrak{p}} Y\right]$. Since $\mathfrak{g}^{\mathbf{c}}$ is simple and $\left[\mathfrak{p}^{\mathbf{c}}, \mathfrak{p}^{\mathbf{c}}\right]+\mathfrak{p}^{\mathbf{c}}$ is an ideal of $\mathfrak{g}^{\mathbf{c}}$, it follows that $\left[\mathfrak{p}^{\mathbf{c}}, \mathfrak{p}^{\mathbf{c}}\right]=\mathfrak{k}^{\mathbf{c}}$. So the issue is to check that $\sigma_{\mathfrak{k}}=f$ on $\mathfrak{k}^{\mathrm{c}}$.

Write $\mathfrak{k}^{\mathrm{c}}=\mathfrak{k}_{\mathrm{ss}}^{\mathrm{c}}+\mathfrak{z}^{\mathrm{c}}$, where $\mathfrak{k}_{\mathrm{ss}}^{\mathrm{c}}$ is the semisimple part of $\mathfrak{k}^{\mathrm{c}}$. By Proposition 2.4, we can ignore the center $\mathfrak{z}^{\mathrm{c}}$ and check (3.4) only on $\mathfrak{k}_{\mathrm{ss}}^{\mathrm{c}}$. If (3.4) holds for some non-zero root vector $[X, Y] \in \mathfrak{k}^{\mathbf{c}}$, then given any other $[Z,[X, Y]] \in \mathfrak{k}_{\mathrm{ss}}^{\mathbf{c}}$ with $Z \in \mathfrak{k}^{\mathbf{c}}$, we have

$$
\begin{aligned}
\sigma_{\mathfrak{k}}[Z,[X, Y]] & =\left[\sigma_{\mathfrak{k}} Z, \sigma_{\mathfrak{k}}[X, Y]\right] \\
& =\left[\sigma_{\mathfrak{k}} Z,\left[\sigma_{\mathfrak{p}} X, \sigma_{\mathfrak{p}} Y\right]\right] \\
& =\left[\left[\sigma_{\mathfrak{k}} Z, \sigma_{\mathfrak{p}} X\right], \sigma_{\mathfrak{p}} Y\right]+\left[\sigma_{\mathfrak{p}} X,\left[\sigma_{\mathfrak{k}} Z, \sigma_{\mathfrak{p}} Y\right]\right] \\
& =\left[\sigma_{\mathfrak{p}}[Z, X], \sigma_{\mathfrak{p}} Y\right]+\left[\sigma_{\mathfrak{p}} X, \sigma_{\mathfrak{p}}[Z, Y]\right] \\
& =f[[Z, X], Y]+f[X,[Z, Y]] \\
& =f[Z,[X, Y]] .
\end{aligned}
$$

Therefore, it suffices to check (3.4) on any one non-zero root vector of $\mathfrak{k}^{\mathrm{c}}$, because it then holds for all $\mathfrak{k}_{\mathrm{ss}}^{\mathrm{c}}$. 
We first check (3.4) for $\mathfrak{g}$ of equal rank non-Hermitian type. After that, with minor modifications, similar arguments also work for $\mathfrak{g}$ of Hermitian type and of non-equal rank type.

Assuming $\mathfrak{g}$ is of equal rank non-Hermitian type, its almost double Vogan diagram has a unique black vertex $\gamma$ with $m_{\gamma}=2$. The diagram leads to an involution $\sigma$ on the root system. Let $\beta$ be a $\mathfrak{k}^{\mathfrak{c}}$-root fixed by $\sigma$. It can be written as

$$
\beta=2 \gamma+\sum_{D_{\mathfrak{k}}} b_{\alpha} \alpha
$$

Since $\sigma \beta=\beta$, we have $b_{\alpha}=b_{\sigma \alpha}$ for all $\alpha$. Hence by letting $b_{\gamma}=2$, we can use the format of (3.2) and rewrite (3.5) as

$$
\beta=\sum_{\overline{\mathcal{O}}} b_{\alpha} \alpha
$$

We can also express $\beta$ with only the white vertices, namely $\beta=\sum_{D_{\mathfrak{k}}} c_{\alpha} \alpha$. Once again $c_{\alpha}=c_{\sigma \alpha}$, so by letting $c_{\gamma}=0$, we obtain

$$
\beta=\sum_{\overline{\mathcal{O}}} c_{\alpha} \alpha
$$

By (3.6) and (3.7), $\sum \overline{\mathcal{O}}\left(b_{\alpha}-c_{\alpha}\right) \alpha=0$. Then

$$
\left.\begin{array}{l}
\sum_{\overline{\mathcal{O}}}\left(b_{\alpha}-c_{\alpha}\right) \alpha=0 \\
b_{\gamma}-c_{\gamma}=2=m_{\gamma} \\
\sum_{\mathrm{D}^{1}} m_{\alpha} \alpha=0
\end{array}\right\} \Longrightarrow b_{\alpha}-c_{\alpha}=m_{\alpha} \text { for all } \alpha \in \overline{\mathcal{O}} .
$$

Let $X$ be a non-zero root vector of $\beta$. By applying Corollary 3.2 to (3.6), we have

$$
f X=(-1)^{b} X, \quad b=\sum_{\mathcal{O}} b_{\alpha} .
$$

Similarly, by applying Corollary 3.2 to (3.7), we have

$$
\sigma_{\mathfrak{k}} X=(-1)^{c} X, c=\sum_{\mathcal{O}} c_{\alpha} .
$$

Hence $\sigma_{\mathfrak{k}} X=f X$ if and only if $b-c$ is even. By (3.8), this is equivalent to $\sum_{\mathcal{O}} m_{\alpha}$ being even. This proves the proposition for $\mathfrak{g}$ of equal rank non-Hermitian type.

Next we consider $\mathfrak{g}$ of Hermitian type. There are two black vertices $\{\gamma, \delta\}$, with $m_{\gamma}=m_{\delta}=1$. We again pick a $\mathfrak{k}^{\mathbf{c}}$-root $\beta$ fixed by $\sigma$. Now (3.5) is replaced by $\beta=\gamma+\delta+\sum_{\mathrm{D}_{\mathfrak{k}}} b_{\alpha} \alpha$. So by letting $b_{\gamma}=b_{\delta}=1$, we obtain (3.6). By expressing $\beta$ in terms of the white vertices, we obtain (3.7), with $c_{\gamma}=c_{\delta}=0$. Similar to (3.8),

$$
\left.\begin{array}{l}
\sum_{\overline{\mathcal{O}}}\left(b_{\alpha}-c_{\alpha}\right) \alpha=0 \\
b_{\gamma}-c_{\gamma}=1=m_{\gamma} \\
b_{\delta}-c_{\delta}=1=m_{\delta} \\
\sum_{\mathrm{D}^{1}} m_{\alpha} \alpha=0
\end{array}\right\} \Longrightarrow b_{\alpha}-c_{\alpha}=m_{\alpha} \text { for all } \alpha \in \overline{\mathcal{O}} .
$$

Let $X$ be a non-zero root vector of $\beta$. By applying Corollary 3.2 to (3.6) and (3.7), we obtain (3.9) and (3.10). By similar arguments, $\sigma_{\mathfrak{k}} X=f X$ if and only if $\sum_{\mathcal{O}} m_{\alpha}$ is even. This proves the proposition for $\mathfrak{g}$ of Hermitian type.

Finally we consider $\mathfrak{g}$ of non-equal rank type. Its almost double Vogan diagram appears on $\mathrm{D}^{2}$. It has a unique black vertex $\gamma$, with $m_{\gamma}=1$. Pick a $\mathfrak{k}^{\mathrm{c}}$-root $\beta$ fixed by $\sigma$, and express it involving $\gamma$. This leads to (3.5) and (3.6), with $b_{\gamma}=2$. 
By expressing $\beta$ with only the white vertices, we again obtain (3.7), with $c_{\gamma}=0$. Similar to (3.8),

$$
\left.\begin{array}{l}
\sum_{\overline{\mathcal{O}}}\left(b_{\alpha}-c_{\alpha}\right) \alpha=0 \\
b_{\gamma}-c_{\gamma}=2=2 m_{\gamma} \\
\sum_{\mathrm{D}^{2}} m_{\alpha} \alpha=0
\end{array}\right\} \Longrightarrow b_{\alpha}-c_{\alpha}=2 m_{\alpha} \text { for all } \alpha \in \overline{\mathcal{O}}
$$

Let $X$ be a non-zero root vector of $\beta$. By applying Corollary 3.2 to (3.6) and (3.7), we again obtain (3.9) and (3.10). Therefore, $\sigma_{\mathfrak{k}} X=f X$ if and only if $b-c$ is even. Since $b_{\alpha}-c_{\alpha}=2 m_{\alpha}$, this is equivalent to $2 \sum_{\mathcal{O}} m_{\alpha}$ being even. This proves the proposition for $\mathfrak{g}$ of non-equal rank type. Certainly $2 \sum_{\mathcal{O}} m_{\alpha}$ is always even, so every almost double Vogan diagram on $\mathrm{D}^{2}$ is a double Vogan diagram.

Proof of Theorem 1.3, By Theorem 2.1 and Proposition 3.3, it follows that every double Vogan diagram represents a locally symmetric pair.

The uniqueness assertion in the theorem statement is trivial. Namely, given a double Vogan diagram, it has already uniquely determined $\mathfrak{k}^{\mathbf{c}} \subset \mathfrak{g}^{\mathbf{c}}$ and $\sigma \in \operatorname{inv}\left(\mathfrak{k}^{\mathbf{c}}\right)$. Furthermore, the conditions on the black vertices determine $\sigma$ on the lowest weight spaces $\mathfrak{p}_{\gamma}^{\mathbf{c}}$, and hence uniquely determine $\sigma$ on $\mathfrak{p}^{\mathbf{c}}$ by $\sigma[X, Y]=[\sigma X, \sigma Y]$ for all the other weight spaces $\left[\mathfrak{k}_{\alpha}^{\mathbf{c}}, \mathfrak{p}_{\gamma}^{\mathbf{c}}\right]=\mathfrak{p}_{\alpha+\gamma}^{\mathbf{c}}$ of $\mathfrak{p}^{\mathbf{c}}$. This proves the uniqueness assertion.

Conversely, suppose that we are given a locally symmetric pair $(\mathfrak{g}, \mathfrak{h})$, where $\mathfrak{h}=\mathfrak{g}^{\sigma}$. From $\mathfrak{g}$, we obtain an affine Vogan diagram. There exists a Cartan involution $\theta$ of $\mathfrak{g}$ which commutes with $\sigma$ [11, Thm. 6.16]. Let $\mathfrak{g}^{\mathbf{c}}=\mathfrak{k}^{\mathbf{c}}+\mathfrak{p}^{\mathbf{c}}$ be the decomposition arising from $\theta$. Then $\sigma$ preserves $\mathfrak{k}^{\mathbf{c}}$ and $\mathfrak{p}^{\mathbf{c}}$. Write $\mathfrak{k}^{\mathbf{c}}=\mathfrak{k}_{\mathrm{sS}}^{\mathbf{c}}+\mathfrak{z}^{\mathbf{c}}$, where $\mathfrak{k}_{\mathrm{ss}}^{\mathrm{c}}=\left[\mathfrak{k}^{\mathrm{c}}, \mathfrak{k}^{\mathbf{c}}\right]$ is semisimple, and $\mathfrak{z}^{\mathbf{c}}$ is the center of $\mathfrak{k}^{\mathrm{c}}$. Then $\sigma$ preserves $\mathfrak{k}_{\mathrm{ss}}^{\mathrm{c}}$. By Corollary 2.2, the restriction of $\sigma$ to $\mathfrak{k}_{\mathrm{ss}}^{\mathrm{c}}$ is represented by a Vogan diagram on $\mathrm{D}_{\mathfrak{k}}$. By Proposition 2.4, $\sigma$ is represented by an almost double Vogan diagram. By Proposition 3.3. we have that $r \sum_{\mathcal{O}} m_{\alpha}$ is even. This proves the theorem.

\section{EXtension of InVOLUtions}

In this section, we consider the extension of $\operatorname{inv}\left(\mathfrak{k}^{\mathbf{c}}\right)$ to $\operatorname{inv}\left(\mathfrak{g}^{\mathbf{c}}\right)$ and prove Corollary 1.4. Z. D. Yan had studied this extension problem, and we find similar results in a Chinese book 13] published by Nankai University. However, our approach is simpler and entirely different.

Proof of Corollary 1.4. We first prove Corollary 1.4(a). The extension of $\sigma$ to $\mathfrak{g}^{\mathbf{c}}$ is determined by what we do to the black vertices of the affine Vogan diagrams.

If there is only one black vertex, then there are at most two extensions, namely to circle or uncircle the black vertex. If there are two black vertices and the diagram involution interchanges them, then there is at most one extension, because we are not allowed to circle or uncircle them. It remains to consider the case of two black vertices $\{\gamma, \delta\}$ fixed by the diagram involution. Here we have four preliminary choices: (i) circle both, (ii) uncircle both, (iii) circle only $\gamma$, (iv) circle only $\delta$. But in view of the parity of $\sum_{\mathcal{O}} m_{\alpha}$ imposed by Definition 1.2, these four preliminary choices cannot be simultaneously allowed. Namely, since $m_{\gamma}=m_{\delta}=1$, if we can perform (i) and (ii), then we cannot perform (iii) and (iv), and vice versa. This proves Corollary 1.4(a). We will illustrate the various possibilities on the extension of $\sigma$ in Example 6.3,

In Corollary 1.4(b), there are two black vertices. Then we can either perform (i) and (ii), or perform (iii) and (iv) as mentioned in Corollary 1.4(a) above. In 
general, we do not know whether the two extensions are conjugate. In Section 5, we will discuss the method to check conjugate extensions.

Finally, we prove Corollary 1.4(c). By Definition 1.2 we obtain a double Vogan diagram regardless of whether the black vertex is circled. These two possibilities produce $\sigma= \pm 1$ on the lowest weight space of the adjoint $\mathfrak{k}^{\mathbf{c}}$-representation on $\mathfrak{p}^{\mathbf{c}}$. The sign of an involution on the extreme weight spaces of a $\mathfrak{k}^{\mathbf{c}}$-representation is an intrinsic property, and so the above two $\mathfrak{g}^{\mathbf{c}}$-involutions are not conjugate to each other. This completes the proof of the corollary.

We point out some early works by Z. D. Yan on automorphisms on $\mathfrak{g}^{\mathbf{c}} 13$, Appendix I]. Yan shows that if $\sigma \in \operatorname{inv}\left(\mathfrak{k}^{\mathbf{c}}\right)$ extends to distinct involutions $\sigma_{1}, \sigma_{2}$ on $\mathfrak{g}^{\mathbf{c}}$, then $\sigma_{2}=\sigma_{1} \theta$. This is parallel to our Corollary 1.4(a), together with our observation that $\sigma_{1}$ and $\sigma_{2}$ differ by the circling of black vertices - namely, they differ by a minus sign on $\mathfrak{p}^{\mathbf{c}}$.

Yan also shows that if $\sigma \in \operatorname{inv}\left(\mathfrak{k}^{\mathbf{c}}\right)$ extends to $\sigma \in \operatorname{aut}\left(\mathfrak{g}^{\mathbf{c}}\right)$, then either $\sigma^{2}=1$ or $\sigma^{2}=\theta$ [13, p. 218]. Our machinery of Vogan diagrams does not reveal automorphisms of order 4 , so in the $\sigma^{2}=\theta$ case, we only show that it does not extend to $\operatorname{inv}\left(\mathfrak{g}^{\mathbf{c}}\right)$. Finally, for the non-equal rank case, the existence of distinct extensions is also mentioned in [13] (p. 212, Thm. 1, p. 225, Thm. 2).

\section{Equivalent Diagrams}

In this section, we study an equivalence relation among the double Vogan diagrams, so that the equivalence classes of diagrams correspond bijectively to the conjugate classes of locally symmetric pairs.

The locally symmetric pairs can be regarded as involutions on $\mathfrak{g}$. Two involutions $\sigma, \tau$ on $\mathfrak{g}$ are said to be conjugate if there exists some $A \in \operatorname{aut}(\mathfrak{g})$ such that $A \sigma A^{-1}=$ $\tau$. In particular, if we can find $A$ to be an inner automorphism, we say that $\sigma, \tau$ are inner conjugate. We say that two double Vogan diagrams are equivalent (resp. inner equivalent) if they represent involutions on $\mathfrak{g}$ which are conjugate (resp. inner conjugate).

We have already used Theorem 2.1 to omit some redundant diagrams in Step 2 of (2.6), though occasionally two diagrams under Theorem 2.1 are still equivalent. However, more importantly, equivalent diagrams may result from Step 3 of (2.6), when we extend $\sigma$ from $\mathfrak{k}^{\mathbf{c}}$ to $\mathfrak{g}^{\mathbf{c}}$. Corollary 1.4 says that there may be two extensions to $\operatorname{inv}\left(\mathfrak{g}^{\mathbf{c}}\right)$, and so we need to check if the two extensions are conjugate.

The method to judge equivalent diagrams was originally introduced in [1, 2, and is used to classify all the equivalence classes of Vogan diagrams [5] and extended Vogan diagrams [6]. We shall use a similar method here. Let $\alpha$ be a circled white vertex in a double Vogan diagram. The following algorithm $F_{\alpha}$ produces another double Vogan diagram and is analogous to [1, Sect. 3.7.1] and [2, Sect. 3.7]:

$$
F_{\alpha}:\left\{\begin{array}{l}
\text { reverse circling conditions of all vertices } \beta \text { adjacent to } \alpha, \text { unless: } \\
\beta=\alpha, \text { or } \beta \text { is a longer root joined to } \alpha \text { by a double edge, } \\
\text { or } \beta \text { is not fixed by the involution. }
\end{array}\right.
$$

Theorem 5.1. Let $\mathrm{V}_{1}, \mathrm{~V}_{2}$ be double Vogan diagrams. They are equivalent if and only if there is a sequence of double Vogan diagrams $\mathrm{U}_{i}$ with

$$
\mathrm{V}_{1}=\mathrm{U}_{1} \longrightarrow \mathrm{U}_{2} \longrightarrow \ldots \longrightarrow \mathrm{U}_{n}=\mathrm{V}_{2},
$$


where each $\mathrm{U}_{i} \longrightarrow \mathrm{U}_{i+1}$ is given by some $F_{\alpha}$ of (5.1) or a diagram automorphism. They are inner equivalent if and only if we can find (5.2) such that each $\mathrm{U}_{i} \longrightarrow \mathrm{U}_{i+1}$ is given by some $F_{\alpha}$ without diagram automorphism.

Proof. Let $\sigma \in \operatorname{inv}\left(\mathfrak{g}^{\mathbf{c}}\right)$. A double Vogan diagram of $\sigma$ is drawn after we choose a $\sigma$-stable simple system $\Pi \subset \Delta \subset\left(H^{0}\right)^{*}$ of a $\sigma$-stable Cartan subalgebra $H^{0}$ of $\mathfrak{k}^{\text {c }}$ in (2.3). We shall consider all $\sigma$-stable simple systems of $\Delta$, so that we obtain all the equivalent double Vogan diagrams.

Let $W$ be the Weyl group of $\mathfrak{k}^{\text {c }}$, namely $W$ is generated by the simple reflections in $\Delta$. We have the semi-direct product

$$
\operatorname{aut}(\Delta)=W \times \operatorname{aut}\left(\mathrm{D}_{\mathfrak{k}}\right),
$$

with normal subgroup $W$ 7, Chap. X-3, Thm. 3.29]. Since aut( $\Delta)$ acts transitively on the simple systems, two Vogan diagrams are equivalent if and only if they represent $\sigma \in \operatorname{inv}\left(\mathfrak{g}^{\mathbf{c}}\right)$ with respect to two $\sigma$-stable simple systems $\Pi$ and $a \Pi$ for some $a \in \operatorname{aut}(\Delta)$.

We now compare the double Vogan diagrams with respect to $\Pi$ and $a \Pi$. If $a \in \operatorname{aut}\left(\mathrm{D}_{\mathfrak{k}}\right)$ and also extends to an automorphism on the affine Vogan diagram, the effect is visually obvious, namely we see a diagram automorphism. It remains to consider the effect when $a \in W$. Recall that $W$ is generated by the reflections $r_{\alpha}$, where $\alpha$ is a simple $\mathfrak{k}^{\mathbf{c}}$-root, $r_{\alpha} \alpha=-\alpha$, and $r_{\alpha}$ fixes $\alpha^{\perp}$. The simple $\mathfrak{k}^{\mathbf{c}}$-roots are exactly the vertices $D_{\mathfrak{k}}$. Therefore, we now consider the effect of $r_{\alpha}$ for white vertices $\alpha$.

If $\alpha$ is not fixed by the diagram involution, then the simple system $r_{\alpha} \Pi$ is no longer $\sigma$-stable, so we do not obtain a double Vogan diagram. So we only consider a white vertex $\alpha$ which is fixed by the diagram involution. In this case it may be circled or uncircled. If $\alpha$ is an uncircled vertex, there is no effect on the diagram, namely $\sigma \in \operatorname{inv}\left(\mathfrak{g}^{\mathbf{c}}\right)$ appears the same under $\Pi$ and $r_{\alpha} \Pi$. If $\alpha$ is a circled vertex, then $F_{\alpha}$ of (5.1) precisely describes the effect of $r_{\alpha}$ on the double Vogan diagram of $\sigma$ [1, Sect. 3.7.1], [2, Sect. 3.7]. Since these $r_{\alpha}$ and the diagram automorphisms generate aut $(\Delta)$, we conclude that two double Vogan diagrams $\mathrm{V}_{1}, \mathrm{~V}_{2}$ are equivalent if and only if they are related by (5.2).

By (2.3), $\Delta \subset\left(H^{0}\right)^{*}$, where $H^{0}$ is a $\sigma$-stable Cartan subalgebra of $\mathfrak{k}^{\mathrm{c}}$. If some $A \in$ aut $\left(\mathfrak{k}^{\mathbf{c}}\right)$ preserves $H^{0}$, then it defines an action $a$ on $\Delta$ by $A \mathfrak{k}_{\alpha}^{\mathbf{c}}=\mathfrak{k}_{a \alpha}^{\mathbf{c}}$. Furthermore, $A$ is an inner automorphism if and only if $a \in W$ [11, Chap.VII, Thm. 7.8]. Therefore, the two double Vogan diagrams with respect to $\Pi$ and $a \Pi$ are inner equivalent if and only if $a \in W$. This proves the second statement of the theorem.

\section{EXAMPLES}

In this section, we give some examples of double Vogan diagrams. Example 6.1 explains how $F_{\alpha}$ of (5.1) corresponds to the reflection $r_{\alpha}$ of a white circled vertex $\alpha$, as indicated in the proof of Theorem [5.1. Example 6.2 emphasizes the difference between equivalent and inner equivalent diagrams. Example 6.3 demonstrates the extension of $\operatorname{inv}\left(\mathfrak{k}^{\mathbf{c}}\right)$ to $\operatorname{inv}\left(\mathfrak{g}^{\mathbf{c}}\right)$ and constructs the double Vogan diagrams. It also applies Theorem 5.1 to check for equivalent diagrams. 


\section{Example 6.1.}

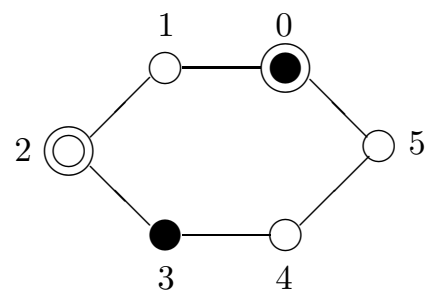

From the two circled vertices, $\sum_{\mathcal{O}} m_{\nu}=2$ is even. By Definition 1.2, this is a double Vogan diagram. By Theorem[1.3, it represents $\sigma \in \operatorname{inv}\left(\mathfrak{g}^{\mathbf{c}}\right)$. We now explain $\sigma$. The labels $0,1, \ldots, 5$ of vertices are used to facilitate the explanation.

The underlying affine Vogan diagram represents $\mathfrak{g}=\mathfrak{s u}(3,3)$. We write the roots as $\left\{\alpha_{i j} ; 1 \leq i, j \leq 6\right.$ and $\left.i \neq j\right\}$ in the usual manner. For $i=1, \ldots, 5$, let vertex $i$ be the simple root $\alpha_{i, i+1}$, so that vertex 0 is the lowest root $\alpha_{61}$. Let $E_{i j}$ be the $6 \times 6$ matrix with 1 at the $(i, j)$-th entry and 0 elsewhere.

The simple root spaces of $\mathfrak{k}^{\mathrm{c}}$ are spanned by $E_{12}, E_{23}, E_{45}, E_{56}$. The involution $\sigma$ acts on $\mathfrak{k}^{\mathrm{c}}$ by $\sigma=1$ on $E_{12}, E_{45}, E_{56}$, and $\sigma=-1$ on $E_{23}$. To extend $\sigma$ to $\mathfrak{g}^{\mathbf{c}}$, we have to circle exactly one of $\{0,3\}$, so that $\sum_{\mathcal{O}} m_{\nu}$ is even. In the above diagram, vertex 3 is not circled, so it forces vertex 0 to be circled. To algebraically see this, we note that when vertex 3 is not circled, then

$$
\begin{aligned}
\sigma E_{16} & =\sigma\left[\left[\left[\left[E_{12}, E_{23}\right], E_{34}\right], E_{45}\right], E_{56}\right] \\
& =\left[\left[\left[\left[\sigma E_{12}, \sigma E_{23}\right], \sigma E_{34}\right], \sigma E_{45}\right], \sigma E_{56}\right] \\
& =\left[\left[\left[\left[E_{12},-E_{23}\right], E_{34}\right], E_{45}\right], E_{56}\right]=-E_{16} .
\end{aligned}
$$

Opposite root spaces have the same sign, so $\sigma E_{61}=-E_{61}$, which forces vertex 0 to be circled.

In the proof of Theorem 5.1, we mention that if $\alpha$ is a circled white vertex, then the simple reflection $r_{\alpha}$ corresponds to the algorithm $F_{\alpha}$ of (5.1). We now illustrate this fact. Let $\alpha$ be vertex 2 . Then

$$
\begin{aligned}
r_{\alpha}\left\{\alpha_{12}, \alpha_{23}, \alpha_{34}, \alpha_{45}, \alpha_{56}, \alpha_{61}\right\} & =\left\{\alpha_{12}+\alpha_{23},-\alpha_{23}, \alpha_{34}+\alpha_{23}, \alpha_{45}, \alpha_{56}, \alpha_{61}\right\} \\
& =\left\{\alpha_{13},-\alpha_{23}, \alpha_{24}, \alpha_{45}, \alpha_{56}, \alpha_{61}\right\} .
\end{aligned}
$$

Note that $\sigma E_{13}=\left[\sigma E_{12}, \sigma E_{23}\right]=\left[E_{12},-E_{23}\right]=-E_{13}$. Similarly, $\sigma E_{24}=-E_{24}$. So if we draw the double Vogan diagram of $\sigma$ with respect to $\left\{\alpha_{13},-\alpha_{23}, \alpha_{24}, \alpha_{45}\right.$, $\left.\alpha_{56}, \alpha_{61}\right\}$, we need to circle vertices $0,1,2,3$. The additional circling of 1,3 caused by $r_{\alpha}$ is precisely algorithm $F_{\alpha}$.

In the proof of Theorem 5.1, we also mention that if $\alpha$ is an uncircled white vertex, there is no effect of $r_{\alpha}$ on the diagram. For example suppose that $\alpha$ is vertex 4 . Then

$$
\begin{aligned}
r_{\alpha}\left\{\alpha_{12}, \alpha_{23}, \alpha_{34}, \alpha_{45}, \alpha_{56}, \alpha_{61}\right\} & =\left\{\alpha_{12}, \alpha_{23}, \alpha_{34}+\alpha_{45},-\alpha_{45}, \alpha_{56}+\alpha_{45}, \alpha_{61}\right\} \\
& =\left\{\alpha_{12}, \alpha_{23}, \alpha_{35},-\alpha_{45}, \alpha_{46}, \alpha_{61}\right\} .
\end{aligned}
$$

Since $\sigma=1$ on the root spaces of $\alpha_{35},-\alpha_{45}, \alpha_{46}$, there is no change on the diagram. 


\section{Example 6.2.}
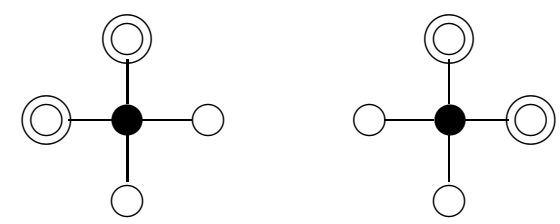

This example serves to highlight the difference between conjugation and inner conjugation. Here each diagram has two circled vertices and $\sum_{\mathcal{O}} m_{\nu}=2$ is even, so they are double Vogan diagrams. By diagram automorphism, it is obvious that they are equivalent. Consequently, they represent conjugate involutions on $\mathfrak{s o}(4,4)$.

If we use just the algorithms $F_{\alpha}$ and without diagram automorphism, we are not able to transform one diagram to another. To see this, let $\alpha$ denote the middle vertex, and let 1,2 denote the top and left vertices respectively. In the left diagram, vertices 1,2 always have the same circling condition (i.e. both circled or both uncircled). This is because the only way to change the circling conditions of 1 and 2 is by applying $F_{\alpha}$, and $F_{\alpha}$ changes their circling conditions simultaneously. By the same argument, we see that in the right diagram, 1 and 2 always have the opposite circling conditions. So without diagram automorphism, there is no way to transform the left diagram to the right diagram. We conclude that they represent involutions which are conjugate but not inner conjugate.

\section{Example 6.3.}

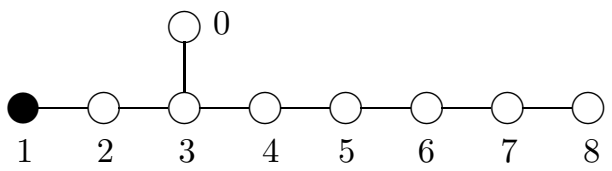

Using Cartan's notation [7 for $\mathfrak{g}$, this is the affine Vogan diagram of $\mathfrak{e}_{8(8)}$, produced by Step 1 of (2.6). We now carefully explain how it gives rise to four nonequivalent double Vogan diagrams.

We now perform Steps 2 and 3 of (2.6). There is no non-trivial diagram involution on $E_{8}^{1}$, so we only need to circle the vertices. Let $\left(i_{1}, \ldots, i_{k}\right)$ denote the circling of vertices $i_{1}, \ldots, i_{k}$.

We shall write down a set $S$ which consists of a representative from each inner equivalence class of Vogan diagrams on $D_{\mathfrak{k}}=D_{8}$. It then suffices to consider the extensions of members of $S$ to $\operatorname{inv}\left(\mathfrak{g}^{\mathbf{c}}\right)$. This is because if a Vogan diagram $\mathrm{U}$ is inner equivalent to $\mathrm{V} \in S$ via some sequence $\left\{F_{\alpha}\right\}_{\alpha}$, and if $\mathrm{U}$ extends to a double Vogan diagram $\tilde{\mathrm{U}}$, then the same sequence $\left\{F_{\alpha}\right\}_{\alpha}$ also transforms $\tilde{\mathrm{U}}$ to an extension of V. By Theorem 2.1, we only need to consider Vogan diagrams on $D_{\mathfrak{k}}$ with a single circling, namely $\{(0),(2),(3), \ldots,(8)\}$. Note that the condition $m_{\alpha}=1,2$ of Theorem 2.1] is irrelevant on $\mathrm{D}_{\mathfrak{k}}=D_{8}$. Here (6) is inner equivalent to (4), and (3) is inner equivalent to (7) 5. There is no particular preference, so we omit (6) and (3) and let $S$ be the following six diagrams:

$$
\begin{array}{ll}
\text { (a) } & (2),(5),(7), \\
\text { (b) } & (0),(4),(8) .
\end{array}
$$


It turns out that (0) and (2) are equivalent but not inner equivalent in $D_{8}$ (this issue has to do with the parity of $n$ in $D_{n}$ ), so we list them both in (6.1).

Since Definition 1.2 requires $\sum_{\mathcal{O}} m_{\alpha}$ to be even, each member of (6.1) (b) does not extend to $\operatorname{inv}\left(\mathfrak{g}^{\mathbf{c}}\right)$. For instance, (0) in (6.1) (b) does not extend because whether or not we circle vertex 1 , we end up with $m_{0}=3$ or $m_{0}+m_{1}=3+2=5$. So we ignore (6.1) (b).

By the same reason, each member of (6.1) (a) extends to $\operatorname{inv}\left(\mathfrak{g}^{\mathbf{c}}\right)$ by circling or uncircling vertex 1 . For instance, $(2)$ extends to $(2),(1,2) \in \operatorname{inv}\left(\mathfrak{g}^{\mathbf{c}}\right)$ because $m_{2}=4$ and $m_{1}+m_{2}=2+4=6$. Therefore, by applying Step 3 of (2.6) to (6.1) (a), we obtain six double Vogan diagrams

$$
(2),(5),(7),(1,2),(1,5),(1,7) \text {. }
$$

Next we look for equivalent diagrams among (6.2). The equivalence relation is defined via $F_{\alpha}$ for white circled vertices $\alpha$, so we cannot apply $F_{1}$ to the above diagrams. Therefore, since (2), (5), (7) are mutually non-equivalent Vogan diagrams on $\mathrm{D}_{\mathfrak{k}}$, they are also non-equivalent as double Vogan diagrams. It remains to see if any of $(1,2),(1,5),(1,7)$ is equivalent to any of $(2),(5),(7)$.

We claim that

$$
(1,5) \sim(5),(1,7) \sim(7) .
$$

To check this, we first transform them to some diagrams which do not circle vertex 1 (because 1 is black), and then use [5, Table 1] to see that their resulting Vogan diagrams on $D_{8}$ are equivalent to (5) and (7), respectively. For instance $F_{5}, F_{4}, F_{3}, F_{2}$ transform $(1,5)$ to $(0,2,6)$; then [5, Table 1$]$ says that $(0,2,6) \sim(5)$. We can also check directly that $F_{5}, F_{4}, F_{3}, F_{2}, F_{0}, F_{3}, F_{4}, F_{5}$ transform $(1,5)$ to $(5)$, and similarly $F_{7}, F_{6}, \ldots, F_{2}, F_{0}, F_{3}, F_{4}, \ldots, F_{7}$ transform $(1,7)$ to $(7)$. This proves (6.3) as claimed.

Finally we show that $(1,2)$ is not equivalent to any of $(2),(5),(7)$. By $[6$, Table 1] on $E_{8}^{1}$, we see that $(1,2)$ cannot be transformed to $(2)$ or $(7)$ by any sequence of $F_{\alpha}$, even when we allow $F_{1}$ in $[6$. So it remains only to show that $(1,2)$ is not equivalent to (5). Since $F_{2}(1,2)=(2,3)$, we can instead compare $(2,3)$ with $(5)$. Both $(2,3)$ and $(5)$ do not circle the black vertex 1 , and as diagrams of $\mathrm{D}_{\mathfrak{k}}$, they are not equivalent 5 . Hence they are also not equivalent double Vogan diagrams. We have thus shown that $(1,2)$ is not equivalent to $(5)$.

We conclude that all the distinct conjugate classes of $\operatorname{inv}\left(\mathfrak{e}_{8(8)}\right)$ are represented by

$$
(2),(5),(7),(1,2) \text {. }
$$

Indeed these four diagrams are shown in the next section.

Recall that by Corollary 1.4(a), a $\mathfrak{k}^{\mathrm{c}}$-involution can have 0,1 or 2 extensions to $\operatorname{inv}\left(\mathfrak{g}^{\mathbf{c}}\right)$, up to conjugation. In this example, the $\mathfrak{k}^{\mathbf{c}}$-involutions in (6.1) (b) have no extension. The $\mathfrak{k}^{\mathbf{c}}$-involution (5) has one extension, because $(5)$ and $(1,5)$ are equivalent. The $\mathfrak{k}^{\mathbf{c}}$-involution (2) has two extensions $(2)$ and $(1,2)$.

\section{Classification of LOCAlly Symmetric Pairs}

In this section, we apply Theorems 1.3 and 5.1 to draw all the double Vogan diagrams other than equivalent ones. They provide an independent classification of the conjugate classes of locally symmetric pairs. For convenience, we decide not to distinguish diagrams which differ by a diagram automorphism, such as those 
in Example 6.2. Consequently, the diagrams do not distinguish locally symmetric pairs which are conjugate but not inner conjugate.

We first explain how we match the diagrams with the locally symmetric pairs $(\mathfrak{g}, \mathfrak{h})$. Two locally symmetric pairs which correspond to the pairs of involutions $(\theta, \sigma)$ and $(\theta, \theta \sigma)$ are called associate pairs. By Corollary 1.4(a), each locally symmetric pair is associated to at most one other pair. Our diagrams effectively detect associate pairs, because they agree on $\mathrm{D}_{\mathfrak{k}}$. Let $\mathrm{T}(\cdot)$ denote the type of a real reductive Lie algebra. For instance $\mathrm{T}(\mathfrak{s u}(3,4))=A$, or more precisely $\mathrm{T}(\mathfrak{s u}(3,4))=A_{6}$. Distinct associate pairs have the same $T(\mathfrak{g}), \mathrm{T}(\mathfrak{k})$ and $\mathrm{T}(\mathfrak{h} \cap \mathfrak{k})$, and different $\mathrm{T}(\mathfrak{h})$.

Recall that $\sim$ denotes conjugate $\mathfrak{g}$-involutions. Now let $\approx$ denote conjugate $\mathfrak{g}^{\mathbf{c}}$ involutions. If $\sigma, \tau \in \operatorname{inv}(\mathfrak{g})$, let $\sigma^{\mathbf{c}}, \tau^{\mathbf{c}} \in \operatorname{inv}\left(\mathfrak{g}^{\mathbf{c}}\right)$ be their complex linear extensions. Clearly $\sigma \sim \tau$ implies $\sigma^{\mathbf{c}} \approx \tau^{\mathbf{c}}$. The condition $\sigma^{\mathbf{c}} \approx \tau^{\mathbf{c}}$ is independent of their behaviors on $\mathfrak{g}$. So $\sigma^{\mathbf{c}} \approx \tau^{\mathbf{c}}$ if and only if their Vogan diagrams are related by (5.1) and (5.2), where $F_{\alpha}$ can be applied so long as $\alpha$ is a circled vertex (regardless of white or black).

Given a double Vogan diagram U, suppose that we want to find $T(\mathfrak{h})$. Apply Theorem 1.1 to U and obtain a circled affine diagram $\mathrm{V}$ (independent of white and black vertices) such that $\mathrm{U} \approx \mathrm{V}$, and $\mathrm{V}$ satisfies

$$
r \sum_{\text {circled }} m_{\alpha}=2
$$

By Theorem 1.1 the uncircled vertices of $\mathrm{V}$ then reveal $\mathrm{T}(\mathfrak{h})$.

Consider Example 6.3, where $\mathfrak{g}=\mathfrak{e}_{8(8)}$. We now match its four double Vogan diagrams in (6.4) with the four locally symmetric pairs $(\mathfrak{g}, \mathfrak{h})$ in [9, pp. 118-119], with $\mathfrak{h}$ given by

$$
\mathfrak{e}_{7(7)}+\mathfrak{s l}(2, \mathbf{R}), \mathfrak{s o}^{*}(16), \mathfrak{e}_{7(-5)}+\mathfrak{s u}(2), \mathfrak{s o}(8,8)
$$

As indicated in [9], the first two cases of (7.2) are associate pairs, while the last two are self-associate. Therefore, the associate diagrams (2) and $(1,2)$ of (6.4) are matched with $\mathfrak{e}_{7(7)}+\mathfrak{s l}(2, \mathbf{R})$ and $\mathfrak{s o}^{*}(16)$. It remains to determine "which is which" in the associate pair, and we check with $\mathrm{T}(\mathfrak{h})$. First consider the diagram (2). Since $m_{2}=4$, it does not satisfy (7.1), so we replace (2) by (7). This is because $(2) \approx(7)$ (by [6, p. 115, Table 1]), and also (7) satisfies (7.1) (because $m_{7}=2$ ). The uncircled vertices of $(7)$ give $\mathrm{T}(\mathfrak{h})=E_{7} \times A_{1}$. Since $\mathrm{T}\left(\mathfrak{e}_{7(7)}+\mathfrak{s l}(2, \mathbf{R})\right)=E_{7} \times A_{1}$ and $\mathrm{T}\left(\mathfrak{s o}^{*}(16)\right)=D_{8}$, we conclude that (2) represents $\mathfrak{h}=\mathfrak{e}_{7(7)}+\mathfrak{s l}(2, \mathbf{R})$. This automatically implies that $(1,2)$ represents $\mathfrak{h}=\mathfrak{s o}^{*}(16)$. Indeed we can verify it similarly: Here $(1,2) \approx(1)$ (by [6, p. 115 , Table 1] or by $F_{1}(1,2)=(1)$ ) and $(1)$ satisfies (7.1). The uncircled vertices of $(1)$ provide $\mathrm{T}(\mathfrak{h})=D_{8}$, which coincides with $\mathrm{T}\left(\mathfrak{s o}^{*}(16)\right)$.

Next we consider the self-associate diagram (5) of (6.4). We replace (5) by (1), because $(5) \approx(1)$ [6, p. 115, Table 1] and (1) satisfies (7.1). The uncircled vertices of (1) reveal $\mathrm{T}(\mathfrak{h})=D_{8}$. So from (7.2), we know that (5) represents $\mathfrak{h}=\mathfrak{s o}(8,8)$. Even though both $\mathfrak{s o}^{*}(16)$ and $\mathfrak{s o}(8,8)$ of (7.2) have type $D_{8}$, we choose $\mathfrak{s o}(8,8)$ because only $\mathfrak{s o}(8,8)$ is self-associate, and also because $\mathfrak{s o}^{*}(16)$ has already been picked by diagram $(1,2)$. 
By similar arguments, we also see that $(7)$ represents $\mathfrak{h}=\mathfrak{e}_{7(-5)}+\mathfrak{s u}(2)$. This matches the diagrams (6.4) of $\mathfrak{e}_{8(8)}$ with the locally symmetric pairs (7.2).

If the diagram involution of $\sigma$ does not preserve the subdiagram $\mathrm{D}$ of $\mathrm{D}^{1}$, we need to look at the second row of its square quadruplet or its dual pair [9]. For instance, consider the following double Vogan diagram, where the diagram involution does not preserve $C_{n}$ of $C_{n}^{1}$.

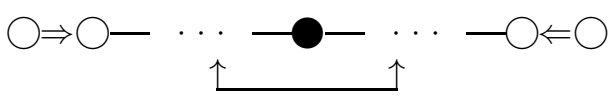

We get two non-conjugate diagrams by circling or uncircling the middle black vertex. We use the square quadruplets

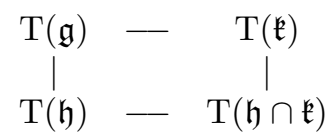

in 9. Quadruplets of associate pairs differ only at the lower left corner, $\mathrm{T}(\mathfrak{h})$. In particular, the two associate quadruplets obtained from the above affine Vogan diagram (by circling the middle black vertex or not) are

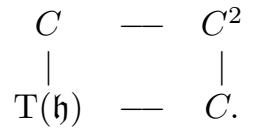

By [9, p. 114, (III-C-1)], we get either $\mathfrak{h}=\mathfrak{g l}(n, \mathbf{H})$ with $\mathrm{T}(\mathfrak{h})=A$, or $\mathfrak{h}=\mathfrak{s p}(n, \mathbf{C})$ with $\mathrm{T}(\mathfrak{h})=C^{2}$. From $\mathfrak{g l}(n, \mathbf{H})$, we know that if the second row of the quadruplet is $A-C$, it should correspond to the diagram with an uncircled fixed vertex. This leaves $\mathfrak{h}=\mathfrak{s p}(n, \mathbf{C})$ for the diagram with a circled black vertex.

Using the methods illustrated above, we now match the diagrams with the locally symmetric pairs. We first list the diagrams on $\mathrm{D}^{1}$, then followed by the diagrams on $\mathrm{D}^{2}$. Each table starts with an affine Dynkin diagram $\mathrm{D}^{r}$ and indicates $\mathfrak{g}$ and $\mathfrak{k}$. It is followed by all the double Vogan diagrams with the prescribed $\mathfrak{g}$.

For the classical Lie algebras $\mathfrak{g}$, we write down the corresponding locally symmetric pairs $\left(\mathfrak{g}, \mathfrak{g}^{\sigma}\right)$. For convenience of the statements, we refer to the classical matrix Lie algebras such as $\mathfrak{g l}(n, \mathbf{R})$ and $\mathfrak{u}(p, q)$ without requiring their traces to be zero. We also omit the special cases of our general diagrams. For example in the first locally symmetric pair $\left(\mathfrak{g}, \mathfrak{g}^{\sigma}\right)=(\mathfrak{u}(k+r, l+s), \mathfrak{u}(k, l)+\mathfrak{u}(r, s))$, we omit the diagram where the circled vertex happens to be black, in which case some of $k, l, r, s$ become zero. We use $\mathfrak{s p}(n, \mathbf{R})$ and $\mathfrak{s p}(n, \mathbf{C})$ to denote the $2 n \times 2 n$ real and complex matrices of type $C_{n}$, unlike the notation $\mathfrak{s p}(2 n, \mathbf{R})$ and $\mathfrak{s p}(2 n, \mathbf{C})$ used in [9, Section 4]. We also make the convention that all vertices which are not shown are the white vertices without circling.

For the exceptional Lie algebras $\mathfrak{g}$, we indicate the corresponding $\mathfrak{h}=\mathfrak{g}^{\sigma}$. We use Cartan's notation (see for example [7, Chap. X-6]) to describe the exceptional real Lie algebras. For instance $\mathfrak{e}_{n(c)}$ is the real Lie algebra of type $E_{n}$, such that $\operatorname{dim} \mathfrak{p}-\operatorname{dim} \mathfrak{k}=c$. 


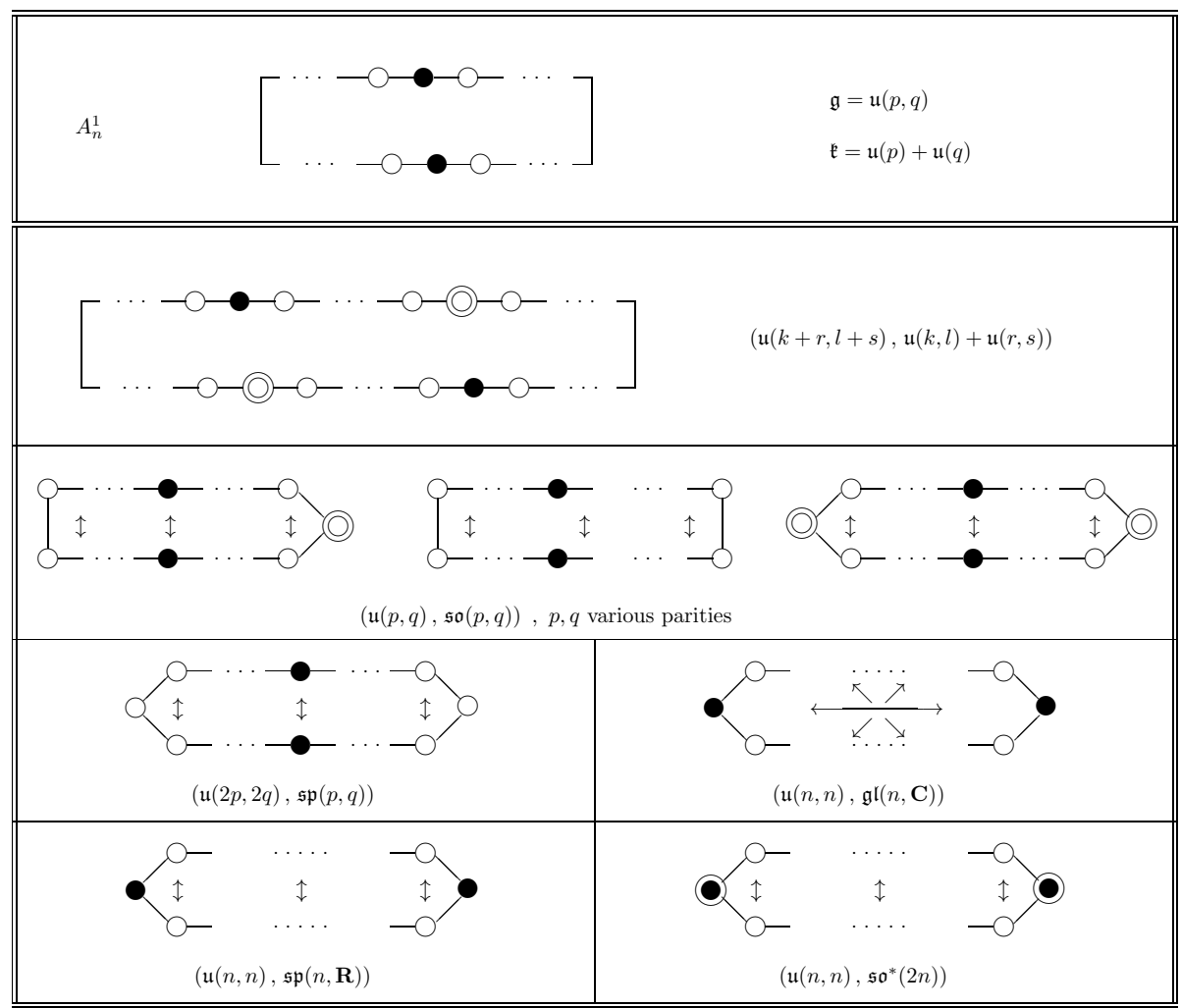

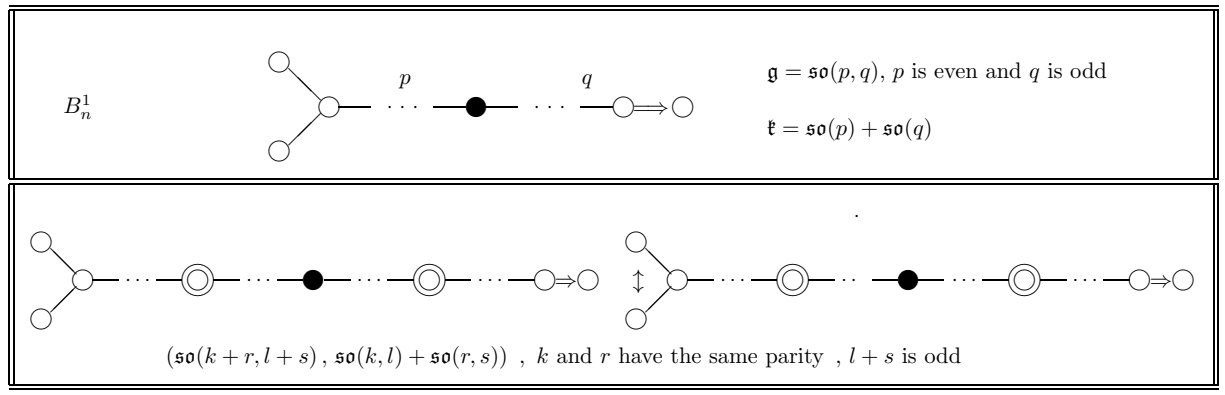




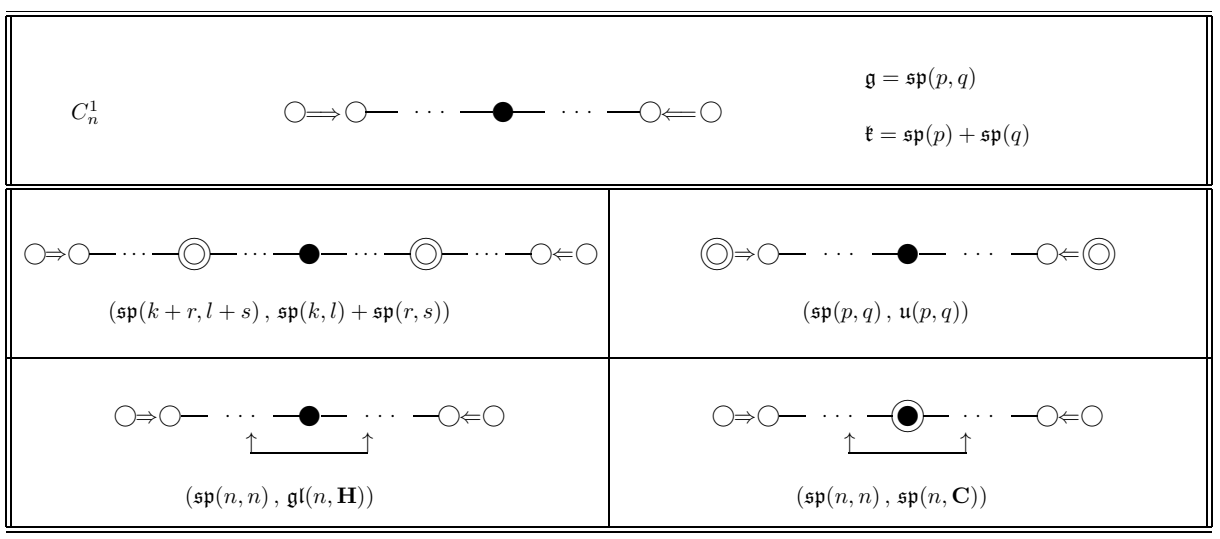

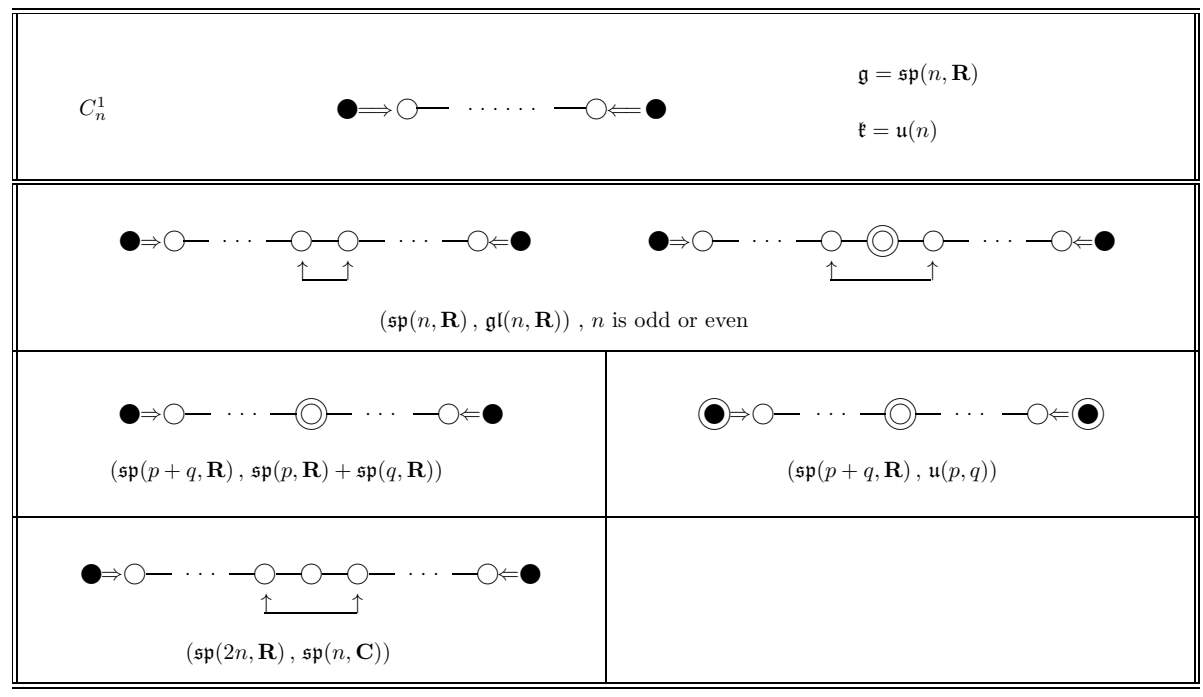




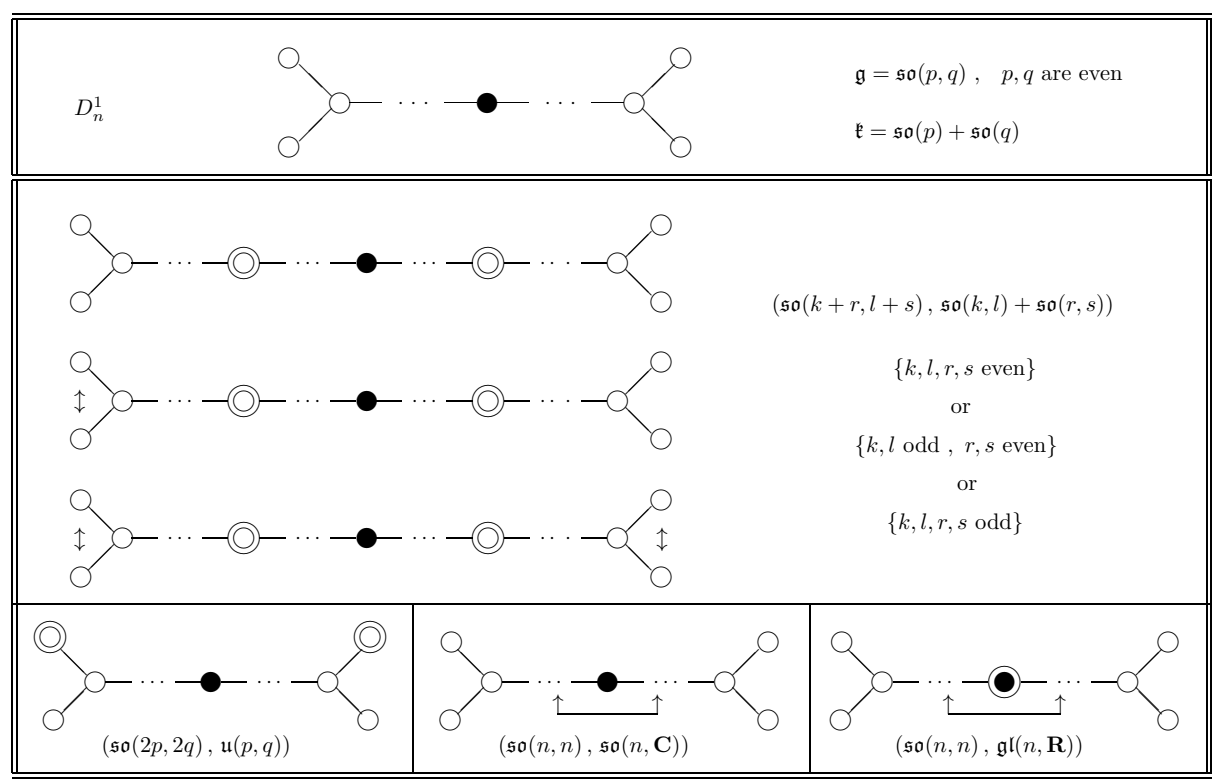

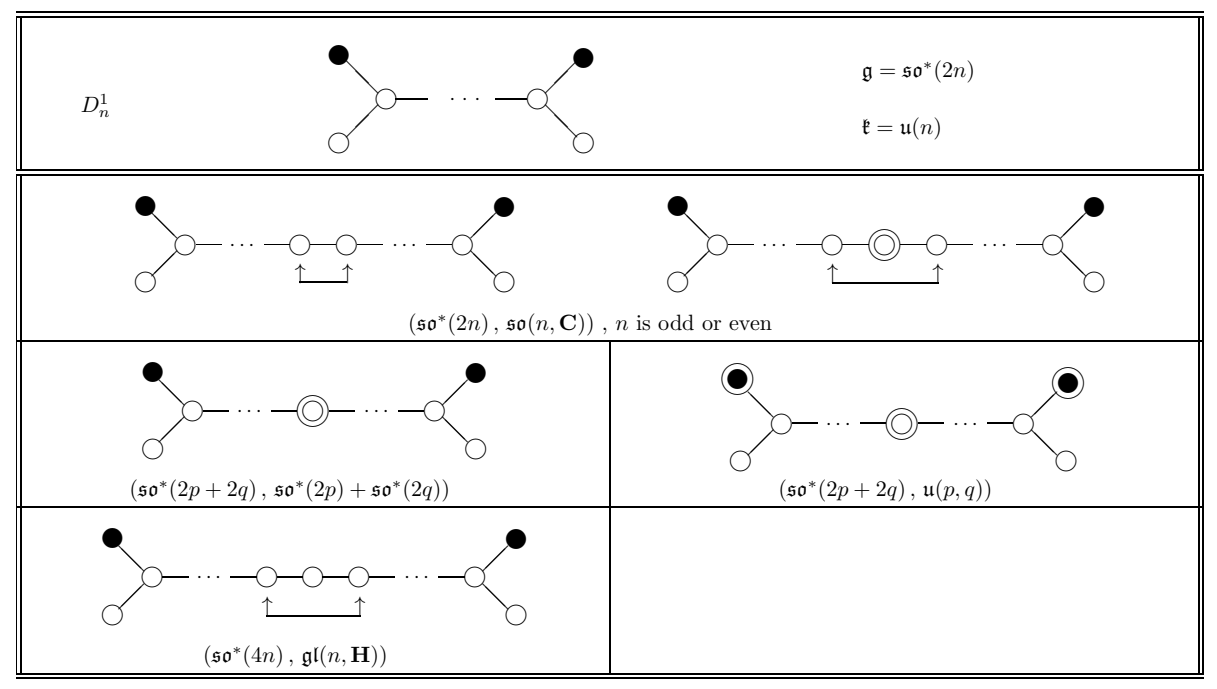



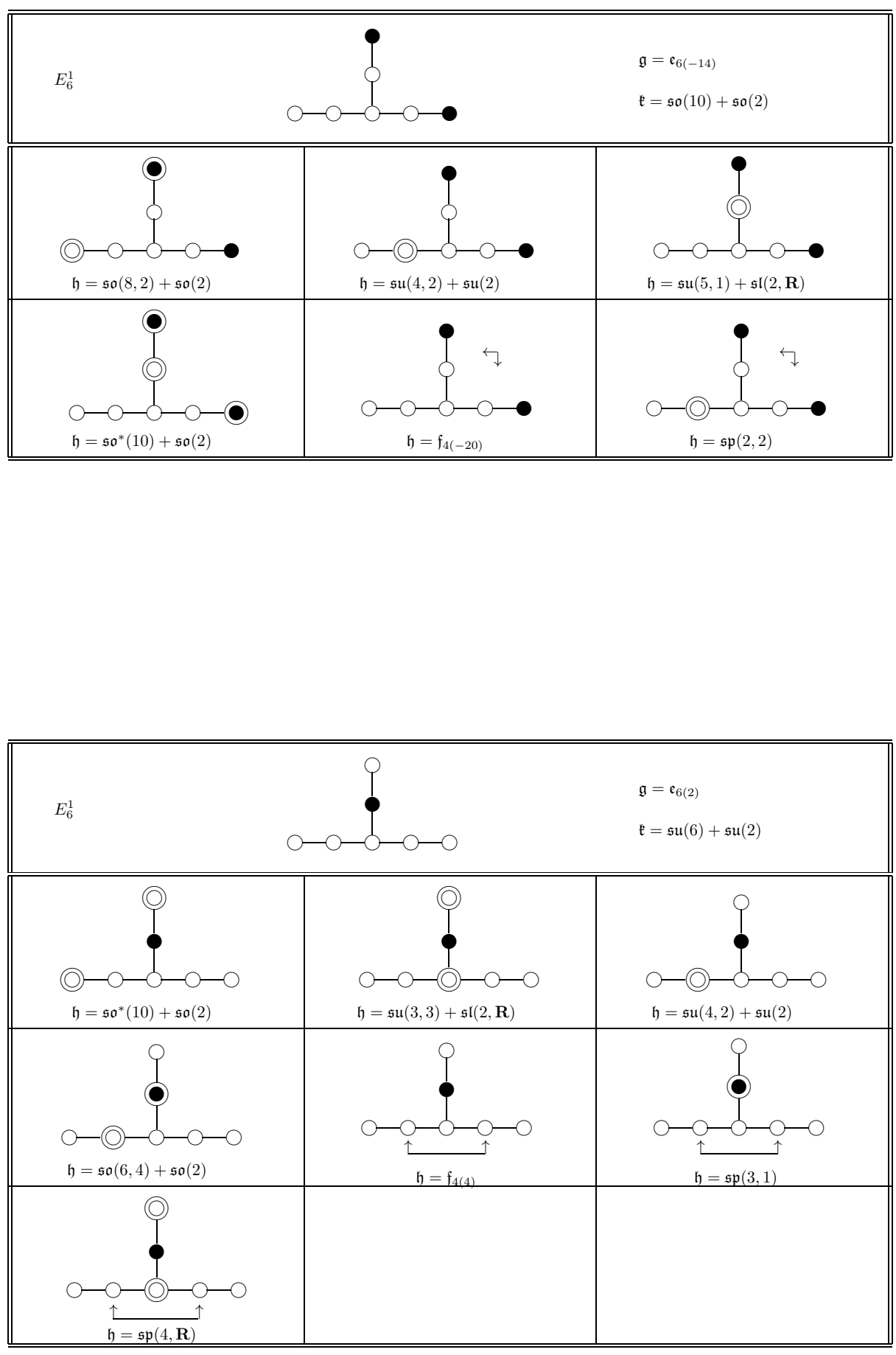

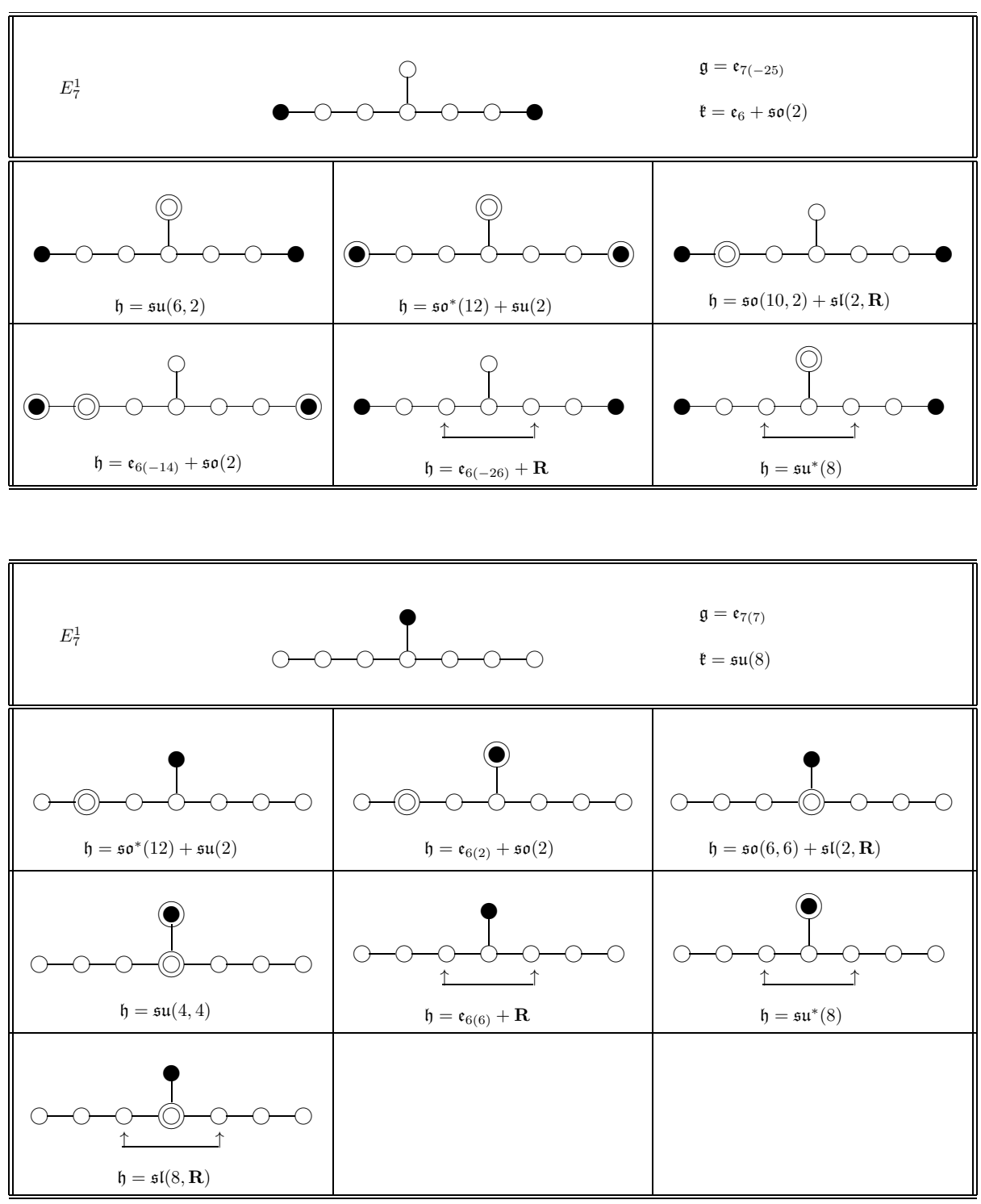

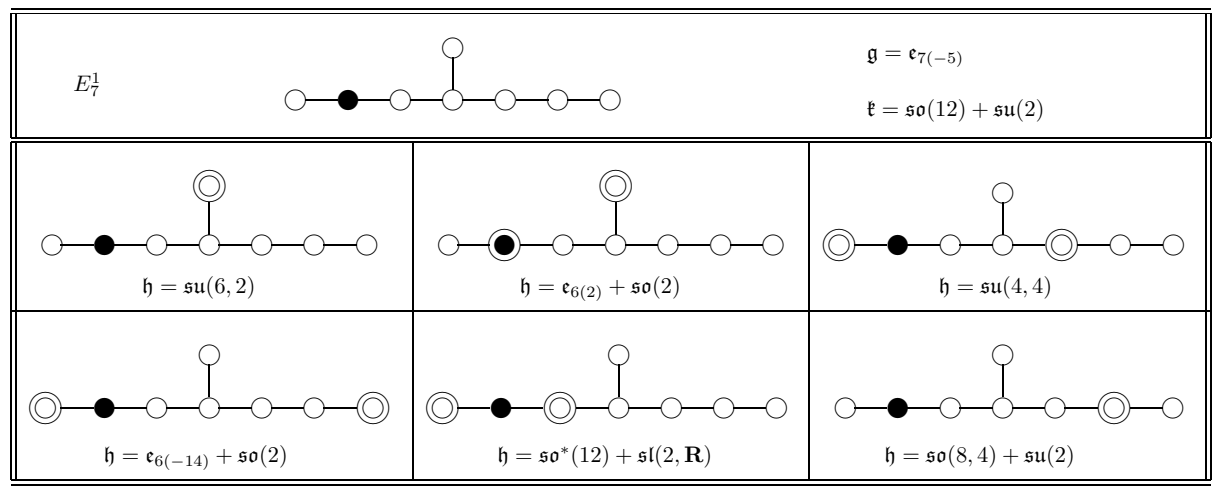




\begin{tabular}{||c|c|c|c||}
\hline \hline \\
\hline
\end{tabular}

\begin{tabular}{||c|c|c|c||}
\hline \hline \\
\hline
\end{tabular}

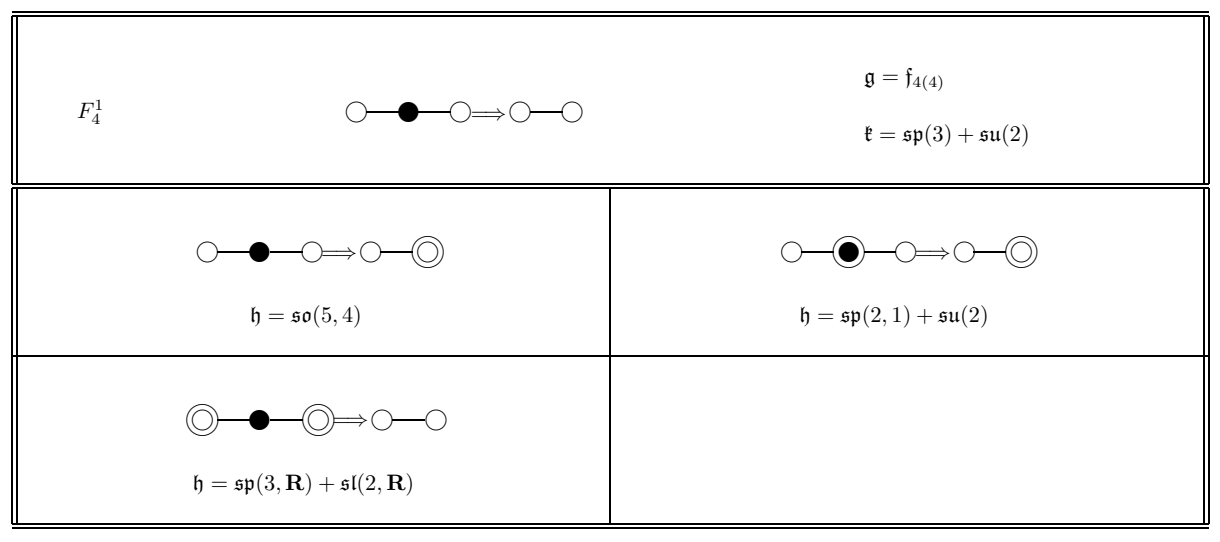

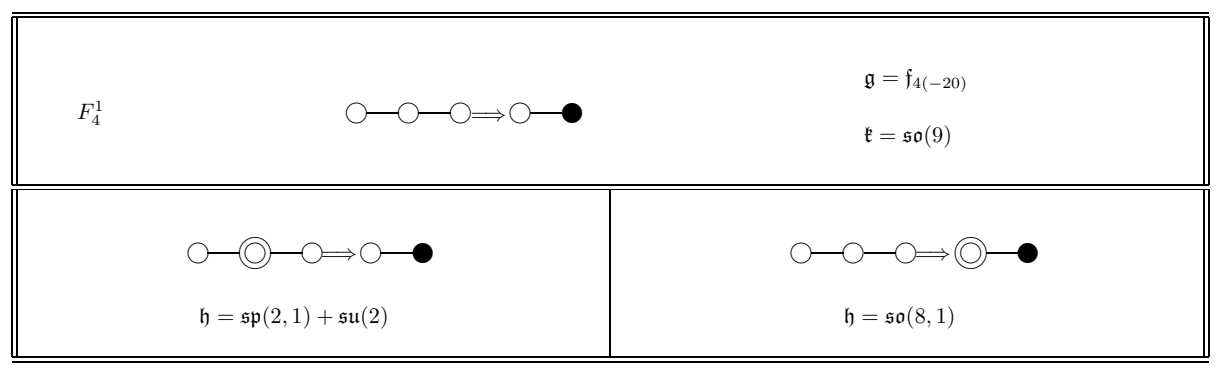




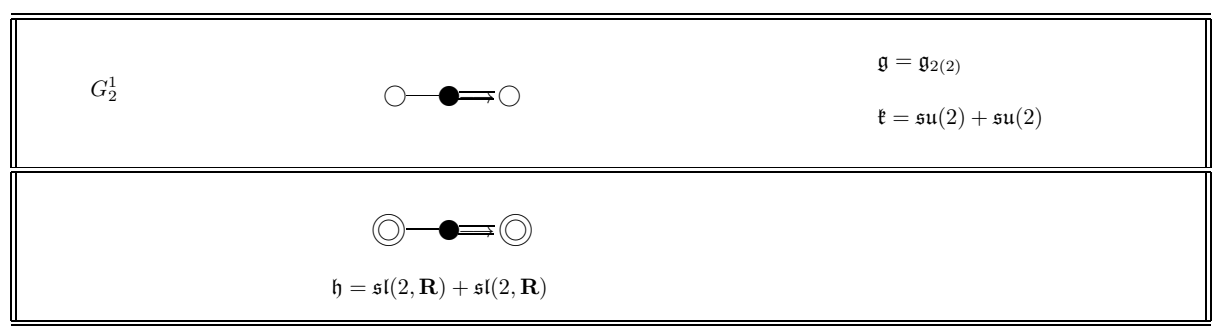

\begin{tabular}{|c|c|}
\hline$\Longrightarrow \bigcirc-\cdots-\bigcirc \Longrightarrow \bigcirc$ & $\begin{array}{l}\mathfrak{g}=\mathfrak{g l}(n, \mathbf{R}), n \text { is odd } \\
\mathfrak{k}=\mathfrak{o}(n)\end{array}$ \\
\hline $\begin{array}{l}\Longrightarrow \bigcirc-\cdots-\cdots \\
(\mathfrak{g l}(p+q, \mathbf{R}), \mathfrak{g l}(p, \mathbf{R})+\mathfrak{g l}(q, \mathbf{R}))\end{array}$ & 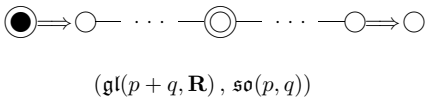 \\
\hline
\end{tabular}

\begin{tabular}{||c|c||}
\hline \hline \\
\hline \hline$(\mathfrak{g l}(p+q, \mathbf{H}), \mathfrak{g l}(p, \mathbf{H})+\mathfrak{g l}(q, \mathbf{H}))$
\end{tabular}

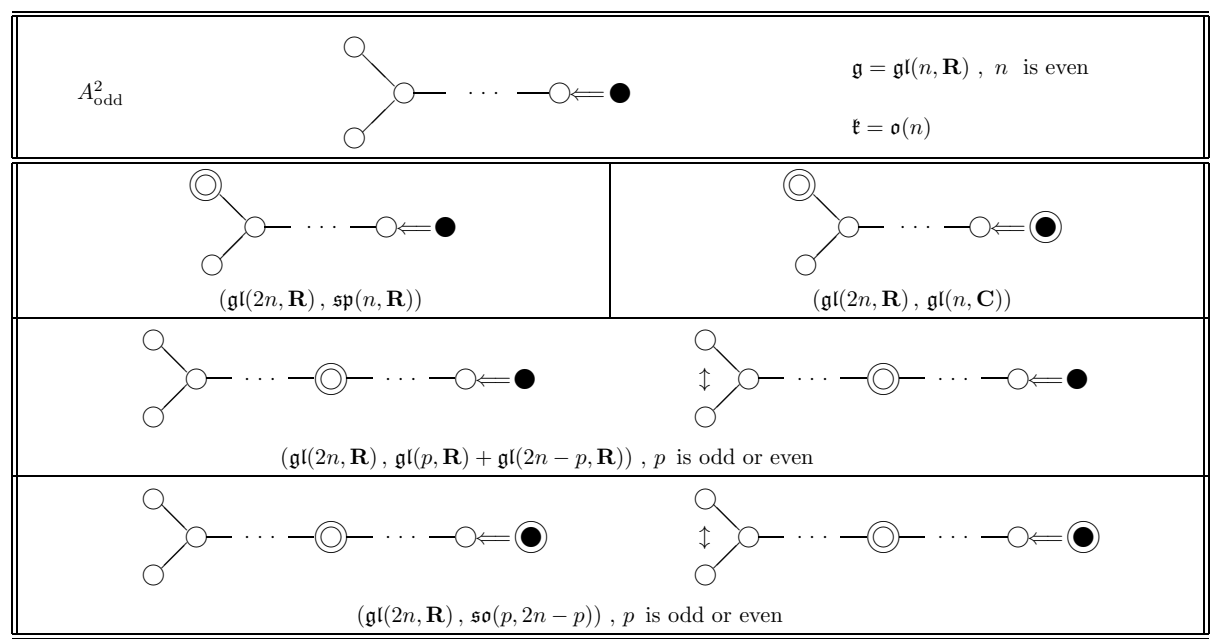




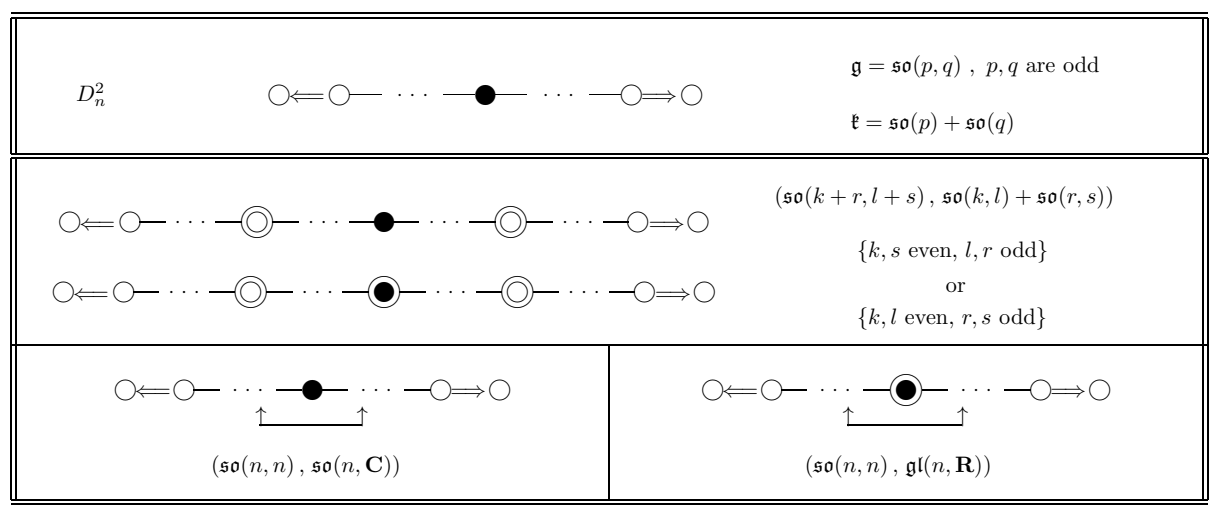

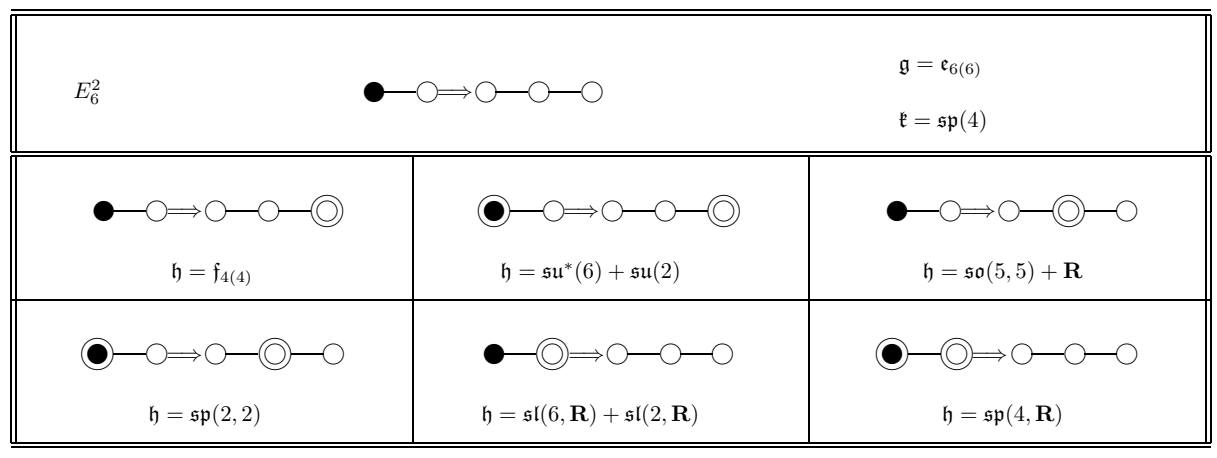

\begin{tabular}{|c|c|c|}
\hline \multicolumn{2}{|c|}{$\mathrm{O}-\mathrm{O} \Longrightarrow \mathrm{O}-\mathrm{O}-\bullet$} & $\begin{array}{l}\mathfrak{g}=\mathfrak{e}_{6}(-26) \\
\mathfrak{k}=\mathfrak{f}_{4}\end{array}$ \\
\hline $\begin{array}{c}\longrightarrow \longrightarrow \mathrm{O} \longrightarrow \mathrm{O} \\
\mathfrak{h}=\mathfrak{s p}(3,1)\end{array}$ & 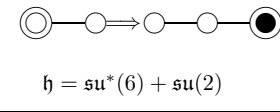 & $\begin{array}{c}\mathrm{O} \Longrightarrow \mathrm{O} \Longrightarrow(\mathrm{O}-\mathrm{C} \\
\mathfrak{h}=\mathfrak{s o}(9,1)+\mathbf{R}\end{array}$ \\
\hline $\begin{array}{c}\mathrm{O} \Longrightarrow \mathrm{O} \longrightarrow \mathrm{O}-\mathrm{C} \\
\mathfrak{h}=\mathfrak{f}_{4(-20)}\end{array}$ & & \\
\hline
\end{tabular}




\section{Appendix: Exceptional LOCALly Symmetric PAirs}

In the previous section, we use some important information of locally symmetric pairs (such as associate pairs) to match them with the double Vogan diagrams. However, for exceptional locally symmetric pairs, the information in 9, pp. 116119] contains some misprints and mistakes. In view of their importance as needed in the previous section, and for the reader's convenience, we take this opportunity to correct the mistakes of [9, pp. 116-119] and present the accurate information here.

Recall that the locally symmetric pairs corresponding to $(\theta, \sigma)$ and $(\theta, \theta \sigma)$ are known as associate pairs. Furthermore, the one corresponding to $(\sigma, \theta)$ is known as the dual pair of the original one. Let $\mathfrak{g}$ be a real exceptional Lie algebra in this section. As before, let $\mathfrak{k}$ and $\mathfrak{h}$ be the fixed points of $\theta$ and $\sigma$, respectively. All the dual pairs and associate pairs have the same type $T(\mathfrak{h} \cap \mathfrak{k})$. Therefore, when we present the locally symmetric pairs in the following groups labelled by $T(\mathfrak{h} \cap \mathfrak{k})$, the dual and associate pairs appear in the same group. In order to be consistent with the notation of [9, pp. 116-119], we let $\mathfrak{l}^{\mathbf{c}}$ denote $\mathrm{T}(\mathfrak{h} \cap \mathfrak{k})$.

I. $\mathfrak{g}^{\mathbf{c}}=E_{6}$. There are 23 pairs.

(I-1) $\quad \mathfrak{l}^{\mathbf{c}}=A_{3}$. The $(\mathfrak{g}, \mathfrak{h})$-pairs are

$$
\begin{aligned}
&\left(\mathfrak{e}_{6(6)}, \mathfrak{s p}(4, \mathbf{R})\right)(\text { self-dual }) \stackrel{\text { asso }^{\prime}}{\longleftrightarrow}\left(\mathfrak{e}_{6(6)}, \mathfrak{s l}(6, \mathbf{R})+\mathfrak{s l}(2, \mathbf{R})\right) \\
& \stackrel{\text { dual }}{\longleftrightarrow}\left(\mathfrak{e}_{6(2)}, \mathfrak{s p}(4, \mathbf{R})\right)(\text { self-asso. }) .
\end{aligned}
$$

(I-2) $\mathfrak{l}^{\mathbf{c}}=B_{2} \times B_{2}$. The $(\mathfrak{g}, \mathfrak{h})$-pairs are

$$
\begin{aligned}
\left(\mathfrak{e}_{6(6)}, \mathfrak{s p}(2,2)\right)(\text { self-dual }) & \stackrel{\text { asso. }}{\longleftrightarrow}\left(\mathfrak{e}_{6(6)}, \mathfrak{s o}(5,5)+\mathbf{R}\right) \\
& \stackrel{\text { dual }}{\longleftrightarrow}\left(\mathfrak{e}_{6(-14)}, \mathfrak{s p}(2,2)\right) \text { (self-asso.). }
\end{aligned}
$$

(I-3) $\quad \mathfrak{l}^{\mathbf{c}}=A_{4}$. The $(\mathfrak{g}, \mathfrak{h})$-pairs are

$$
\begin{aligned}
\left(\mathfrak{e}_{6(-14)}, \mathfrak{s o}^{*}(10)+\mathfrak{s o}(2)\right)(\text { self-dual }) & \stackrel{\text { asso. }}{\longleftrightarrow}\left(\mathfrak{e}_{6(-14)}, \mathfrak{s u}(5,1)+\mathfrak{s l}(2, \mathbf{R})\right) \\
& \left.\stackrel{\text { dual }}{\longleftrightarrow}\left(\mathfrak{e}_{6(2)}, \mathfrak{s o}^{*}(10)+\mathfrak{s o}(2)\right) \text { (self-asso. }\right) .
\end{aligned}
$$

(I-4) $\mathfrak{l}^{\mathbf{c}}=A_{3} \times A_{1} \times A_{1}$. The $(\mathfrak{g}, \mathfrak{h})$-pairs are

$$
\begin{aligned}
\left(\mathfrak{e}_{6(-14)}, \mathfrak{s u}(4,2)+\mathfrak{s u}(2)\right)(\text { self-asso. }) & \stackrel{\text { dual }}{\longleftrightarrow}\left(\mathfrak{e}_{6(2)}, \mathfrak{s o}(6,4)+\mathfrak{s o}(2)\right) \\
& \stackrel{\text { asso. }}{\longleftrightarrow}\left(\mathfrak{e}_{6(2)}, \mathfrak{s u}(4,2)+\mathfrak{s u}(2)\right) \text { (self-dual). }
\end{aligned}
$$

(I-5) $\mathfrak{l}^{\mathbf{c}}=A_{2} \times A_{2}$. The $(\mathfrak{g}, \mathfrak{h})$-pair is

$$
\left(\mathfrak{e}_{6(2)}, \mathfrak{s u}(3,3)+\mathfrak{s l}(2, \mathbf{R})\right)(\text { self-dual and self-asso. }) \text {. }
$$

(I-6) $\quad \mathfrak{l}^{\mathfrak{c}}=B_{4}$. The $(\mathfrak{g}, \mathfrak{h})$-pairs are

$$
\begin{aligned}
\left(\mathfrak{e}_{6(-14)}, \mathfrak{f}_{4(-20)}\right)(\text { self-asso. }) & \stackrel{\text { dual }}{\longleftrightarrow}\left(\mathfrak{e}_{6(-26)}, \mathfrak{s o}(9,1)+\mathbf{R}\right) \\
& \stackrel{\text { asso. }}{\longleftrightarrow}\left(\mathfrak{e}_{6(-26)}, \mathfrak{f}_{4(-20)}\right) \text { (self-dual). }
\end{aligned}
$$

(I-7) $\quad \mathfrak{l}^{\mathbf{c}}=D_{4}$. The $(\mathfrak{g}, \mathfrak{h})$-pair is

$$
\left(\mathfrak{e}_{6(-14)}, \mathfrak{s o}(8,2)+\mathfrak{s o}(2)\right)(\text { self-dual and self-asso.). }
$$

(I-8) $\mathfrak{l}^{\mathbf{c}}=C_{3} \times A_{1}$. The $(\mathfrak{g}, \mathfrak{h})$-pairs are

$$
\begin{array}{cccc}
\left(\mathfrak{e}_{6}(-26), \mathfrak{s u ^ { * }}(6)+\mathfrak{s u}(2)\right) & \stackrel{\text { dual }}{\longleftrightarrow}\left(\mathfrak{e}_{6(2)}, \mathfrak{f}_{4(4)}\right) & \stackrel{\text { asso. }}{\longleftrightarrow} & \left(\mathfrak{e}_{6(2)}, \mathfrak{s p}(3,1)\right) \\
& & & \uparrow \text { dual } \\
\left(\mathfrak{e}_{6(-26)}, \mathfrak{s p}(3,1)\right) & \stackrel{\text { dual }}{\longleftrightarrow}\left(\mathfrak{e}_{6(6)}, \mathfrak{f}_{4(4)}\right) & \stackrel{\text { asso. }}{\longleftrightarrow} & \left(\mathfrak{e}_{6(6)}, \mathfrak{s u}^{*}(6)+\mathfrak{s u}(2)\right) .
\end{array}
$$


II. $\mathfrak{g}^{\mathbf{c}}=E_{7}$. There are 19 pairs.

(II-1) $\quad \mathfrak{l}^{\mathbf{c}}=A_{5} \times A_{1}$. The $(\mathfrak{g}, \mathfrak{h})$-pairs are

$$
\begin{array}{cccc}
\left(\mathfrak{e}_{7(-5)}, \mathfrak{s u}(6,2)\right) & \stackrel{\text { dual }}{\longleftrightarrow}\left(\mathfrak{e}_{7(7)}, \mathfrak{s o}^{*}(12)+\mathfrak{s u}(2)\right) & \stackrel{\text { asso. }}{\longleftrightarrow} & \left(\mathfrak{e}_{7(7)}, \mathfrak{e}_{6(2)}+\mathfrak{s o}(2)\right) \\
\begin{array}{c}
\uparrow \text { asso. } \\
\left(\mathfrak{e}_{7(-5)}, \mathfrak{e}_{6(2)}+\mathfrak{s o}(2)\right)
\end{array} & \stackrel{\text { dual }}{\longleftrightarrow}\left(\mathfrak{e}_{7(-25)}, \mathfrak{s o}^{*}(12)+\mathfrak{s u}(2)\right) & \stackrel{\text { asso. }}{\longleftrightarrow} & \left(\mathfrak{e}_{7(-25)}, \mathfrak{s u}(6,2)\right) .
\end{array}
$$

$\left(\right.$ II-2) $\quad \mathfrak{l}^{\mathfrak{c}}=A_{3} \times A_{3}$. The $(\mathfrak{g}, \mathfrak{h})$-pairs are

$$
\begin{aligned}
& \left(\mathfrak{e}_{7(-5)}, \mathfrak{s u}(4,4)\right) \text { (self-asso.) } \stackrel{\text { dual }}{\longleftrightarrow}\left(\mathfrak{e}_{7(7)}, \mathfrak{s o}(6,6)+\mathfrak{s l}(2, \mathbf{R})\right) \\
& \stackrel{\text { asso }}{\longleftrightarrow}\left(\mathfrak{e}_{7(7)}, \mathfrak{s u}(4,4)\right) \text { (self-dual). }
\end{aligned}
$$

(II-3) $\quad \mathfrak{l}^{\mathbf{c}}=C_{4}$. The $(\mathfrak{g}, \mathfrak{h})$-pairs are

$$
\begin{aligned}
& \left(\mathfrak{e}_{7(7)}, \mathfrak{s u}^{*}(8)\right)(\text { self-dual }) \stackrel{\text { asso }}{\longleftrightarrow}\left(\mathfrak{e}_{7(7)}, \mathfrak{e}_{6(6)}+\mathbf{R}\right) \\
& \stackrel{\text { dual }}{\longleftrightarrow}\left(\mathfrak{e}_{7(-25)}, \mathfrak{s u}^{*}(8)\right) \text { (self-asso.). }
\end{aligned}
$$

(II-4) $\quad \mathfrak{l}^{\mathbf{c}}=D_{5}$. The $(\mathfrak{g}, \mathfrak{h})$-pairs are

$$
\left(\mathfrak{e}_{7(-5)}, \mathfrak{e}_{6(-14)}+\mathfrak{s o}(2)\right) \text { (self-asso.) } \stackrel{\stackrel{\text { dual }}{\longleftrightarrow}\left(\mathfrak{e}_{7(-25)}, \mathfrak{s o}(10,2)+\mathfrak{s l}(2, \mathbf{R})\right)}{\stackrel{\text { asso }}{\longleftrightarrow}\left(\mathfrak{e}_{7(-25)}, \mathfrak{e}_{6(-14)}+\mathfrak{s o}(2)\right) \text { (self-dual). }}
$$

(II-5) $\quad \mathfrak{l}^{\mathbf{c}}=D_{4} \times A_{1} \times A_{1} \times A_{1}$. The $(\mathfrak{g}, \mathfrak{h})$-pair is

$$
\left(\mathfrak{e}_{7(-5)}, \mathfrak{s o}(8,4)+\mathfrak{s u}(2)\right) \text { (self-dual and self-asso.). }
$$

(II-6) $\quad \mathfrak{l}^{\mathbf{c}}=A_{5}$. The $(\mathfrak{g}, \mathfrak{h})$-pair is

$$
\left(\mathfrak{e}_{7(-5)}, \mathfrak{s o}^{*}(12)+\mathfrak{s l}(2, \mathbf{R})\right)(\text { self-dual and self-asso. }) \text {. }
$$

(II-7) $\quad \mathfrak{l}^{\mathrm{c}}=D_{4}$. The $(\mathfrak{g}, \mathfrak{h})$-pair is

$$
\left(\mathfrak{e}_{7(7)}, \mathfrak{s l}(8, \mathbf{R})\right) \text { (self-dual and self-asso.). }
$$

(II-8) $\quad \mathfrak{l}^{\mathfrak{c}}=F_{4}$. The $(\mathfrak{g}, \mathfrak{h})$-pair is

$$
\left(\mathfrak{e}_{7(-25)}, \mathfrak{e}_{6(-26)}+\mathbf{R}\right) \text { (self-dual and self-asso.). }
$$

III. $\mathfrak{g}^{\mathbf{c}}=E_{8}$. There are 8 pairs.

(III-1) $\quad \mathfrak{l}^{\mathfrak{c}}=D_{6} \times A_{1} \times A_{1}$. The $(\mathfrak{g}, \mathfrak{h})$-pairs are

$$
\begin{aligned}
& \left(\mathfrak{e}_{8(8)}, \mathfrak{e}_{7(-5)}+\mathfrak{s u}(2)\right) \text { (self-asso.) } \stackrel{\text { dual }}{\longleftrightarrow}\left(\mathfrak{e}_{8(-24)}, \mathfrak{s o}(12,4)\right) \\
& \stackrel{\text { asso }}{\longleftrightarrow}\left(\mathfrak{e}_{8(-24)}, \mathfrak{e}_{7(-5)}+\mathfrak{s u}(2)\right) \text { (self-dual). }
\end{aligned}
$$

(III-2) $\quad \mathfrak{l}^{\mathbf{c}}=A_{7}$. The $(\mathfrak{g}, \mathfrak{h})$-pairs are

$$
\begin{aligned}
&\left.\left(\mathfrak{e}_{8(-24)}, \mathfrak{s o}^{*}(16)\right) \text { (self-asso.) }\right) \stackrel{\text { dual }}{\longleftrightarrow}\left(\mathfrak{e}_{8(8)}, \mathfrak{e}_{7(7)}+\mathfrak{s l}(2, \mathbf{R})\right) \\
& \stackrel{\text { asso }}{\longleftrightarrow}\left(\mathfrak{e}_{8(8)}, \mathfrak{s o}^{*}(16)\right)(\text { self-dual }) .
\end{aligned}
$$

(III-3) $\quad \mathfrak{l}^{\mathfrak{c}}=D_{4} \times D_{4}$. The $(\mathfrak{g}, \mathfrak{h})$-pair is

$$
\left(\mathfrak{e}_{8(8)}, \mathfrak{s o}(8,8)\right) \text { (self-dual and self-asso.). }
$$

(III-4) $\quad \mathfrak{l}^{\mathfrak{c}}=E_{6}$. The $(\mathfrak{g}, \mathfrak{h})$-pair is

$$
\left(\mathfrak{e}_{8(-24)}, \mathfrak{e}_{7(-25)}+\mathfrak{s l}(2, \mathbf{R})\right) \text { (self-dual and self-asso.). }
$$

IV. $\mathfrak{g}^{\mathbf{c}}=F_{4}$. There are 5 pairs.

$(\mathbf{I V}-1) \quad \mathfrak{l}^{\mathbf{c}}=B_{2} \times A_{1} \times A_{1}$. The $(\mathfrak{g}, \mathfrak{h})$-pairs are

$$
\left(\mathfrak{f}_{4(-20)}, \mathfrak{s p}(2,1)+\mathfrak{s u}(2)\right) \text { (self-asso.) } \stackrel{\stackrel{\text { dual }}{\longleftrightarrow}\left(\mathfrak{f}_{4(4)}, \mathfrak{s o}(5,4)\right)}{\stackrel{\text { asso. }}{\longleftrightarrow}\left(\mathfrak{f}_{4(4)}, \mathfrak{s p}(2,1)+\mathfrak{s u}(2)\right) \text { (self-dual). }}
$$


$(\mathbf{I V - 2}) \quad \mathfrak{l}^{\mathbf{c}}=D_{4}$. The $(\mathfrak{g}, \mathfrak{h})$-pair is

$$
\left(\mathfrak{f}_{4(-20)}, \mathfrak{s o}(8,1)\right) \text { (self-dual and self-asso.). }
$$

$(\mathbf{I V}-3) \quad \mathfrak{l}^{\mathfrak{c}}=A_{2}$. The $(\mathfrak{g}, \mathfrak{h})$-pair is

$$
\left(\mathfrak{f}_{4(4)}, \mathfrak{s p}(3, \mathbf{R})+\mathfrak{s l}(2, \mathbf{R})\right) \text { (self-dual and self-asso.). }
$$

V. $\mathfrak{g}^{\mathbf{c}}=G_{2}$. The only $(\mathfrak{g}, \mathfrak{h})$-pair is

$$
\left(\mathfrak{g}_{2(-2)}, \mathfrak{s l}(2, \mathbf{R})+\mathfrak{s l}(2, \mathbf{R})\right)(\text { self-dual and self-asso. }) \text {. }
$$

\section{ACKNOWLEDGEMENTS}

We are grateful to Reyer Sjamaar and David Vogan for many helpful conversations. We would also like to thank the referee for the insightful comments, which helped us improve the original manuscript significantly.

\section{REFERENCES}

[1] P. Batra, Invariants of real forms of affine Kac-Moody Lie algebras, J. Algebra 223 (2000), 208-236. MR 1738260 (2001g:17033)

[2] P. Batra, Vogan diagrams of real forms of affine Kac-Moody Lie algebras, J. Algebra 251 (2002), 80-97. MR1900276 (2003d:17027)

[3] M. Berger, Les espaces symétriques non compacts, Ann. Sci. École Norm. Sup. (3) 74 (1957), 85-177. MR0104763 (21:3516)

[4] A. Borel and J. de Siebenthal, Les sous-groupes fermés de rang maximum des groupes de Lie clos, Comment. Math. Helv. 23 (1949), 200-221. MR0032659 (11:326d)

[5] M. K. Chuah and C. C. Hu, Equivalence classes of Vogan diagrams, J. Algebra 279 (2004), 22-37. MR2078384 (2005g:17021)

[6] M. K. Chuah and C. C. Hu, Extended Vogan diagrams, J. Algebra 301 (2006), 112-147. MR.2230323 (2007d:17034)

[7] S. Helgason, Differential Geometry, Lie Groups, and Symmetric Spaces, Graduate Studies in Math. vol. 34, Amer. Math. Soc., Providence, RI, 2001. MR.1834454 (2002b:53081)

[8] A. Helminck, Algebraic groups with a commuting pair of involutions and semisimple symmetric spaces, Advances in Math. 71 (1988), 21-91. MR960363 (90a:17011)

[9] J. S. Huang, Admissible square quadruplets and semisimple symmetric spaces, Advances in Math. 165 (2002), 101-123. MR.1880323 (2003c:53070)

[10] V. Kac, Automorphisms of finite order of semisimple Lie algebras, Funkcional Anal. i Prilozen 3 (1969), 94-96. MR0251091 (40:4322)

[11] A. Knapp, Lie Groups Beyond an Introduction, 2nd. ed., Progr. Math. vol. 140, Birkhäuser, Boston, 2002. MR $1920389(2003 \mathrm{c}: 22001)$

[12] T. Oshima and J. Sekiguchi, The restricted root system of a semisimple symmetric pair, in "Group Representations and Systems of Differential Equations", Advanced Studies in Pure Math. 4 (1984), 433-497. MR810638 (87c:17017)

[13] Z. D. Yan, Real Semisimple Lie Algebras (in Chinese), Nankai Univ. Press, 1998.

Department of Mathematics, National Tsing Hua University, Hsinchu, Taiwan

E-mail address: chuah@math.nthu.edu.tw

Department of Mathematics, The Hong Kong University of Science and Technology, Clear Water Bay, Kowloon, Hong Kong, China

E-mail address: mahuang@ust.hk 